\title{
Performance Demonstration Program Plan for Nondestructive Assay of Boxed Wastes for the TRU Waste Characterization Program
}

Revision 2

October 2009

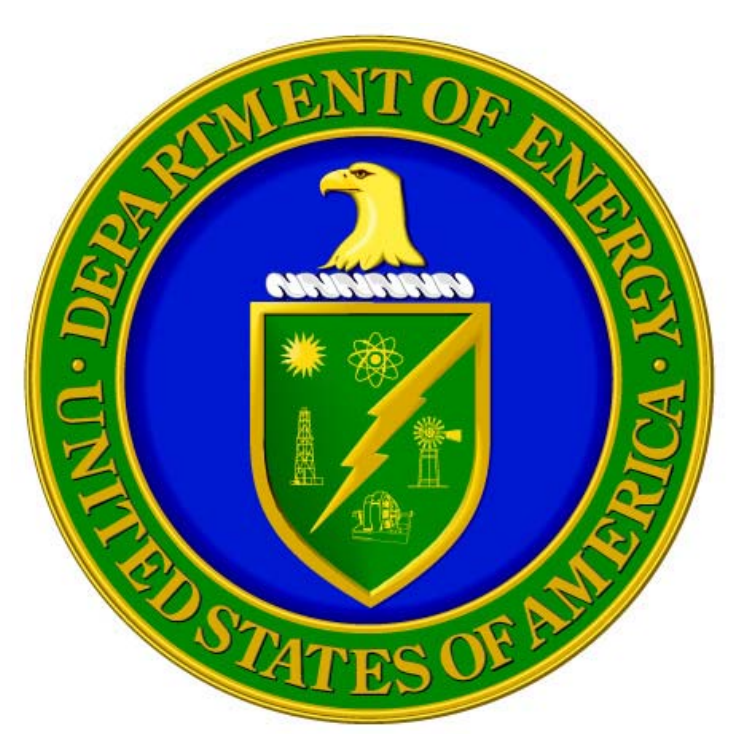

This document supersedes Revision 1 of DOE/CBFO-01-1006

\section{U.S. Department of Energy}

Carlsbad Field Office

Office of the National TRU Program 
This document has been submitted as required to:

Office of Scientific and Technical Information

P.O. Box 62

Oak Ridge, TN 37831

(865) 576-8401

Additional information about this document may be obtained by calling the WIPP Information Center at 1-800-336-9477. Copies may be obtained by contacting the National Technical Information Service, U.S. Department of Commerce, 5285 Port Royal Road, Springfield, VA 22101. 


\title{
Performance Demonstration Program Plan for Nondestructive Assay of Boxed Wastes for the TRU Waste Characterization Program
}

\author{
DOE/CBFO-01-1006 \\ Revision 2 \\ October 2009
}

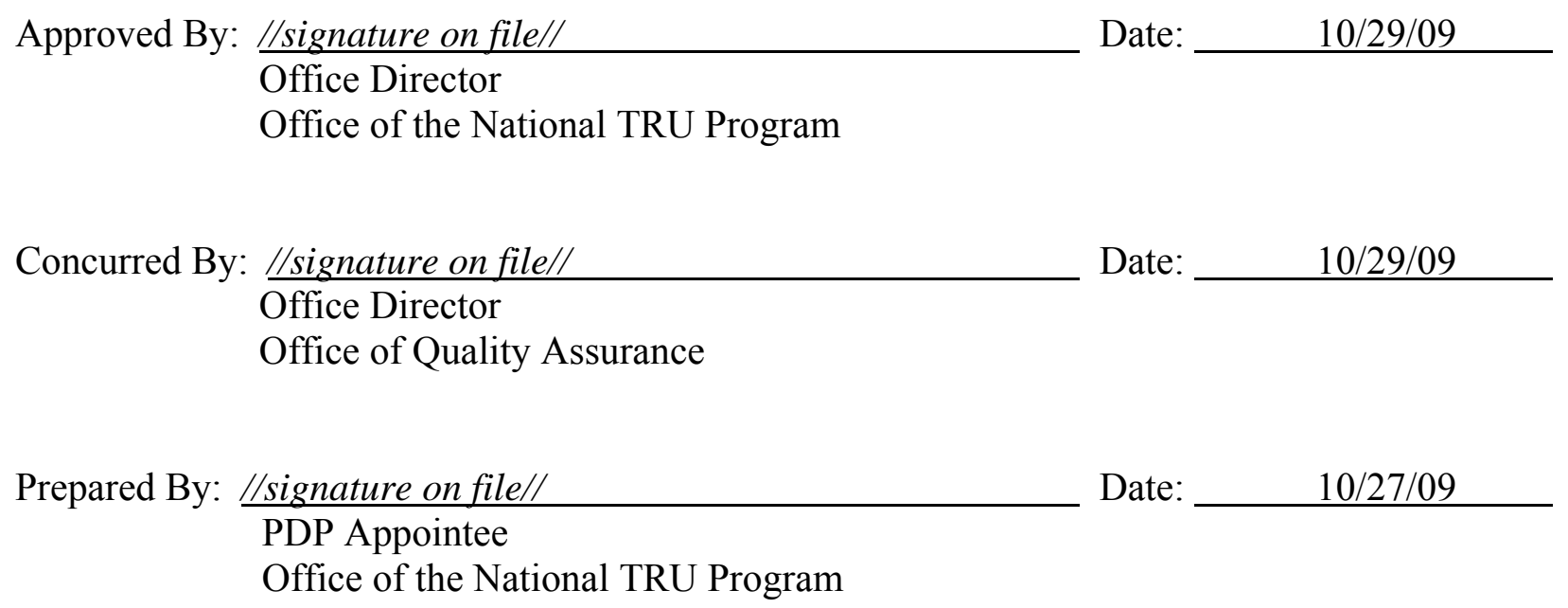


This page intentionally blank 


\section{TABLE OF CONTENTS}

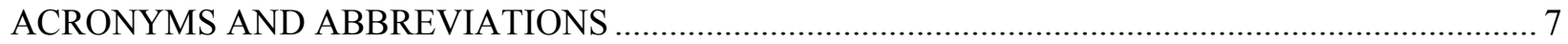

SIGNIFICANT CHANGES TO THE NDA BOX PDP PLAN, REVISION 2 ....................................... 8

1.0 INTRODUCTION TO THE PERFORMANCE DEMONSTRATION PROGRAM ....................... 9

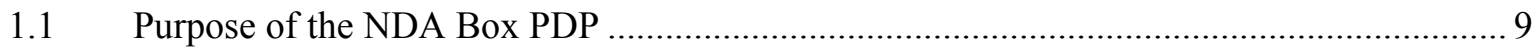

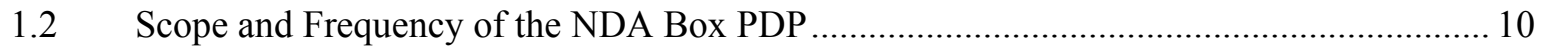

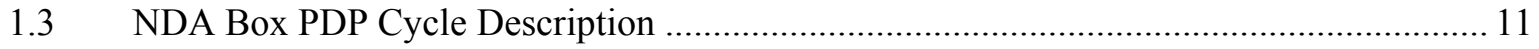

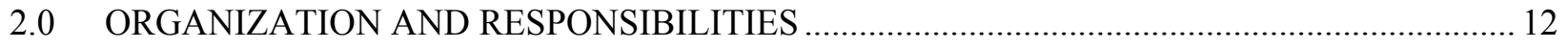

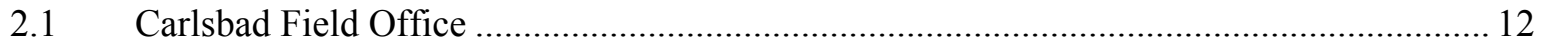

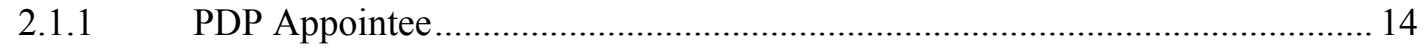

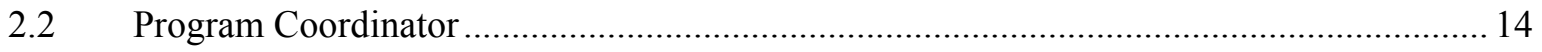

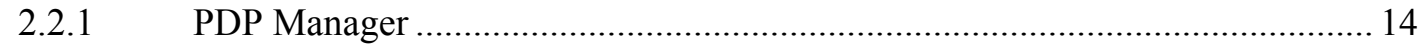

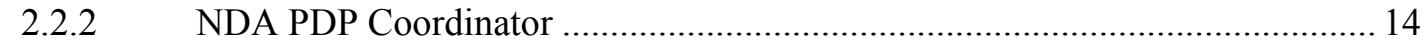

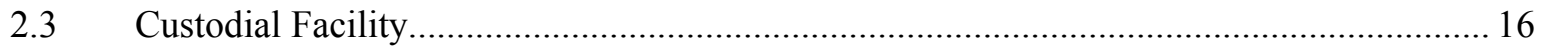

2.3.1 Custodial Facility Assay Coordinator (CFAC) ….......................................... 17

2.3.2 Sample Components Custodian ……........................................................... 17

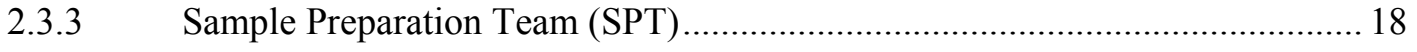

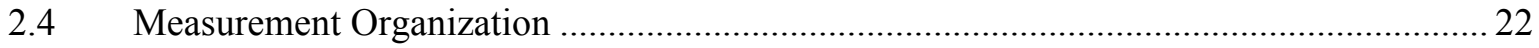

2.4.1 Measurement Organization Assay Coordinator (MOAC) …............................. 22

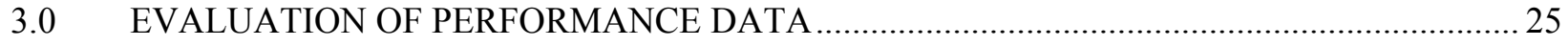

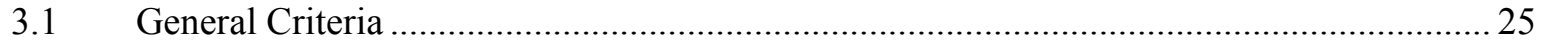

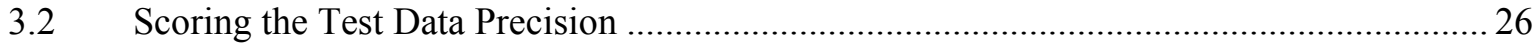

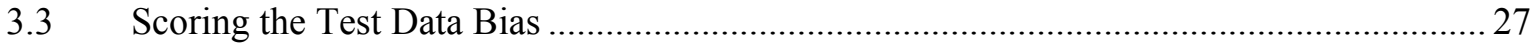

4.0 WIPP QUALIFICATION BASED ON NDA PDP TEST PERFORMANCE .............................28

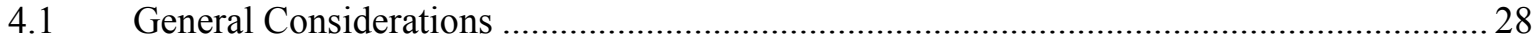

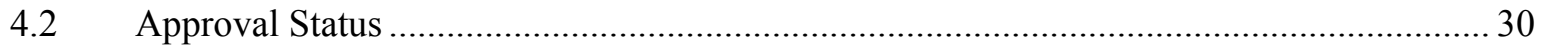

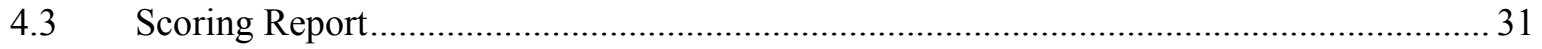

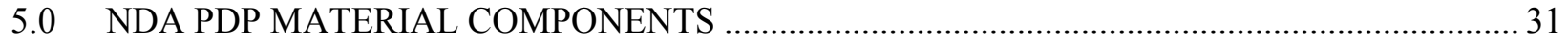

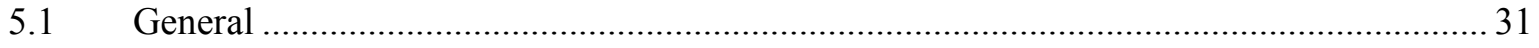

$5.2 \quad$ NTP Working Reference Material (WRM) Standards of the NDA PDP …...................... 32

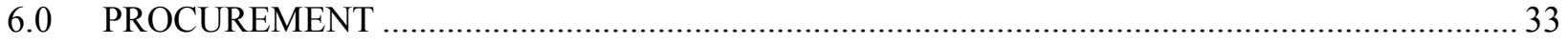

$7.0 \quad$ TRAINING

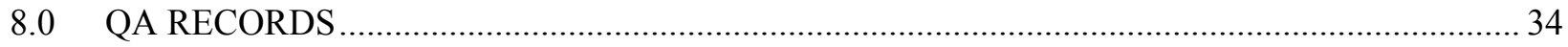




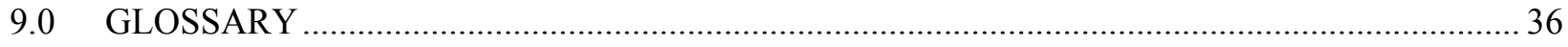

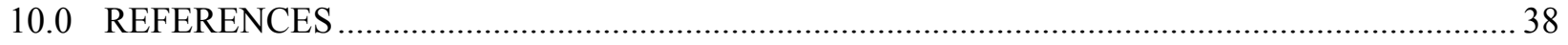

Appendix A NDA Performance Demonstration Program Forms ....................................................... 40

Appendix B Shipment, Receipt, and Use of NDA Box PDP Materials ..............................................50

Appendix C NDA Box PDP System Registration Form ................................................................ 54

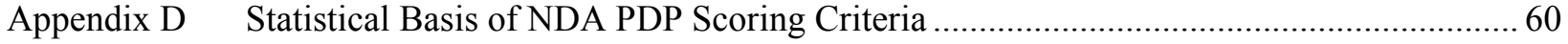

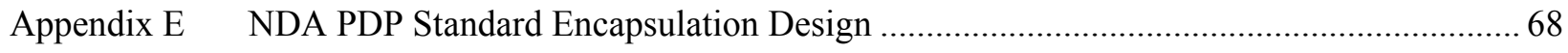

Appendix F NDA PDP Matrix SWB Specifications and Descriptions …......................................... 74 


\section{ACRONYMS AND ABBREVIATIONS}

$\begin{array}{ll}\text { \%R } & \text { percent recovery } \\ \text { \%RSD } & \text { percent relative standard deviation } \\ \text { ASNM } & \text { approval status notification memorandum } \\ \text { CBFO } & \text { Carlsbad Field Office } \\ \text { CFAC } & \text { custodial facility assay coordinator } \\ \text { CH } & \text { contact handled } \\ \text { CTAC } & \text { CBFO Technical Assistance Contractor } \\ \text { DOE } & \text { U.S. Department of Energy } \\ \text { EA } & \text { expert analyst } \\ \text { FGE } & \text { fissile gram equivalent } \\ \text { MDC } & \text { minimum detectable concentration } \\ \text { MOAC } & \text { measurement organization assay coordinator } \\ \text { MP } & \text { management procedure } \\ \text { NDA } & \text { nondestructive assay } \\ \text { NTP } & \text { Office of the National TRU Program } \\ \text { PDP } & \text { Performance Demonstration Program } \\ \text { QA } & \text { quality assurance } \\ \text { QAPD } & \text { Quality Assurance Program Document } \\ \text { RCRA } & \text { Resource Conservation and Recovery Act } \\ \text { SNM } & \text { special nuclear material } \\ \text { SOP } & \text { standard operating procedure } \\ \text { SPT } & \text { sample preparation team } \\ \text { SWB } & \text { standard waste box } \\ \text { TID } & \text { tamper indicating device } \\ \text { TRU } & \text { Transuranic } \\ \text { TWBIR } & \text { Waste Acceptance Criteria } \\ \text { WAC } & \text { Weapons grade plutonium } \\ \text { WIsolation Pilot Plant }\end{array}$




\section{SIGNIFICANT CHANGES TO THE NDA BOX PDP PLAN, REVISION 2}

This document supersedes Revision 1 of the Performance Demonstration Program Plan for Nondestructive Assay of Boxed Wastes for the TRU Waste Characterization Program (DOE/CBFO-01-1006), and is a complete rewrite of that document. This revision constitutes a complete revision of each page; therefore, no change bars are included.

The document was rewritten to:

1. Reorganize the format to emphasize and consolidate cycle participant responsibilities.

2. Better define the process to address failures of the tested NDA systems to meet NDA PDP criteria.

3. Single out the non-interfering matrix SWB as distinct from the other matrix SWBs and to define its use for specialized circumstances.

4. Place information that is not relevant to normal NDA PDP cycle activities in the appendices. 


\subsection{INTRODUCTION TO THE PERFORMANCE DEMONSTRATION PROGRAM}

Each testing and analytical facility performing waste characterization activities for the Waste Isolation Pilot Plant (WIPP) participates in the Performance Demonstration Program (PDP) to comply with the Transuranic Waste Acceptance Criteria for the Waste Isolation Pilot Plant (WAC) (DOE/WIPP-02-3122) and the Quality Assurance Program Document (QAPD) (CBFO94-1012). The PDP serves as a quality control check for data generated in the characterization of waste destined for WIPP. Single-blind audit samples are prepared and distributed to each of the facilities participating in the PDP. Different PDPs evaluate the analyses of simulated headspace gases (HSGs), constituents of the Resource Conservation and Recovery Act (RCRA), and transuranic (TRU) radionuclides using nondestructive assay (NDA) techniques.

The Carlsbad Field Office (CBFO) of the Office of the National TRU Program (NTP) is the review and approval authority for all PDP activities. The CBFO oversees the PDP as described in the current revisions of the following plans:

- DOE/CBFO-01-1005, Performance Demonstration Program Plan for Nondestructive Assay of Drummed Wastes for the TRU Waste Characterization Program (NDA Drum PDP Plan)

- DOE/CBFO-95-1076, Performance Demonstration Program Plan for Analysis of Simulated Headspace Gases (HSG PDP Plan)

- DOE/CBFO-95-1077, Performance Demonstration Program Plan for RCRA Constituent Analysis of Solidified Wastes (RCRA PDP Plan)

- DOE/CBFO-01-1006, Performance Demonstration Program Plan for Nondestructive Assay of Boxed Wastes for the TRU Waste Characterization Program (NDA Box PDP Plan)

This plan, the NDA Box PDP Plan, specifically addresses the purpose, design, management, coordination and conduct of the NDA PDP that applies to contact-handled $(\mathrm{CH})$ TRU waste packaged in standard waste boxes (SWBs).

\subsection{Purpose of the NDA Box PDP}

The NDA Box PDP is designed to test participating measurement organizations' NDA systems (neutron and gamma radiation detection and analysis systems) by evaluating TRU alpha-emitting radionuclide data from NDA measurements of simulated contact-handled $(\mathrm{CH})$ TRU waste. This plan does not address calorimetry, another NDA technique utilized for WIPP waste characterization. The relevant $\mathrm{CH}-\mathrm{TRU}$ wastes addressed by this plan are those generated and packaged in SWBs within the U. S. Department of Energy (DOE) complex. The NDA PDP tests provide a mechanism for the independent and objective assessment of NDA system performance and capability relative to the radiological characterization objectives and criteria of the NTP.

The nuclear material type, mass, and associated alpha activity of the NDA PDP radioactive standards have been specified and fabricated to allow assembly of NDA Box PDP samples that simulate TRU alpha activity concentrations, radionuclide/isotope distributions, and physical 
forms typical of the DOE TRU waste inventory. The PDP waste matrix types were derived from an evaluation of information contained in the Transuranic Waste Baseline Inventory Report (TWBIR) (DOE/CAO-95-1121).

In addition to the NDA PDP, the CBFO approval process includes technical evaluation of the NDA measurement systems through method and performance data supplied by the measurement organization and independent quality assurance audits. Qualified SWB radioassay required by the WIPP may only be performed by measurement organizations and systems that have successfully participated in the NDA Box PDP, meeting the performance criteria in the NDA Box PDP Plan, passing an audit, and being certified by CBFO.

\subsection{Scope and Frequency of the NDA Box PDP}

The CBFO uses the NDA PDP as part of the assessment and approval process for measurement organizations and specific NDA systems characterizing waste for the NTP. Measurement organizations are required to analyze the NDA Box PDP samples using the same procedures approved and implemented for routine operational WIPP waste characterization activities. The NDA PDP is not designed for testing method or procedural changes unless those changes have been accepted by CBFO based upon approved protocol for demonstration of the method validity for the targeted wastes. The NDA PDP is designed to annually "spot check" capabilities for radioassay of one or more specific combinations of matrix type/activity type/activity level/and activity distribution typical of boxed waste being assayed at a specific facility.

This NDA Box PDP Plan describes the elements comprising the test program, including test apparatus, test sample configuration, and required sample analyses. The Plan further identifies and defines the responsibilities of the program entities, including the NDA PDP Coordinator, custodial facility, the facility assay coordinator, the sample preparation team (SPT), and the measurement organization.

All participating facility measurement organizations and NDA systems must demonstrate acceptable performance in the NDA Box PDP before the SWB waste analyses can be WIPPcertified. Systems that have not obtained CBFO approval may choose to proceed with analyses on an "at risk" basis. The organization should recognize that data obtained prior to CBFO approval of the measurement organization, NDA systems, and procedures may be found to be unacceptable to $\mathrm{CBFO}$.

After the initial PDP approval process, each measurement organization and NDA system shall be evaluated annually, as specified in the WAC.

In addition to the primary test cycle, the NDA PDP Coordinator may schedule a supplemental cycle prior to the next primary cycle. A supplemental NDA PDP cycle can be requested by measurement organizations for systems that have failed one or more NDA PDP samples, are newly implemented systems, have been inactive, or have undergone changes that could impact NDA PDP approval status. Requests for supplemental cycles must be submitted in writing to the $\mathrm{CBFO}$ for evaluation. Timing and selection of measurement systems for participation in supplemental cycles will be at the discretion of the CBFO. The CBFO will normally require the 
costs associated with administration of supplemental cycles to be borne by the requesting organization via funds transfer to the PDP Program Coordinator parent contractor.

\subsection{NDA Box PDP Cycle Description}

The NDA Box PDP cycle is a sequence of documented activities designed to produce, evaluate, and report NDA measurement results. The scored results are used as evidence indicating the organization's NDA system and procedural capabilities to produce data of sufficient quality for WIPP certification.

Sequentially, the NDA PDP cycle is described by the following major elements:

- Prior to cycle initiation, the NDA PDP Coordinator identifies the facilities and measurement organizations that will be participating in the cycle and prepares a letter for the CBFO PDP Appointee's signature documenting cycle participants.

- Instructions are sent to the participating facilities' Custodial Facility Assay Coordinators (CFACs) for the assembly and verification of the cycle's PDP samples (activity type, position and quantity, and matrix module type(s) and positioning). These instructions are confidential information; to ensure cycle integrity, these instructions must not be shared with the measurement organization.

- The participating measurement organizations perform nondestructive radioassay of each sample utilizing the NDA systems and procedures authorized by CBFO on a cycle-specific basis. Each sample is counted six (6) times. The results are sent to the NDA PDP Coordinator.

- The NDA PDP Coordinator scores the results against criteria set forth in the NDA Box PDP Plan.

- The NDA PDP Coordinator makes NDA system approval recommendations to the CBFO PDP Appointee based on performance of each of the participating NDA systems to NDA PDP criteria.

- The CBFO PDP Appointee combines the recommendations of the NDA PDP Coordinator with other quality assurance information gathered from onsite assessments to approve, conditionally approve, or disapprove of each of the participating NDA systems on an individual basis.

- If not approved or conditionally approved, a measurement organization may enter a corrective action process to obtain an approved status from CBFO for one or more of their NDA system(s).

- A scoring report is generated that includes: the values reported by the measurement facilities; the reference activity values; the acceptance ranges; the pass-fail status of each individual measurement system relative to the NDA PDP pass-fail criteria; and the CBFO approval status of each participating NDA system. The report is generated 
by the NDA PDP Coordinator and distributed to the WIPP radioassay stakeholders on behalf of the CBFO.

An NDA PDP cycle is considered initiated on the date specified in the letter transmitting the NDA PDP sample configuration instructions to the CFAC. A cycle is concluded upon issuance of the scoring report for the cycle. For compliance with required annual participation, an NDA system must participate in a PDP cycle within 12 months (plus a one-month grace period) from the date results for the cycle are received by the NDA PDP Coordinator (see section 4.2).

\subsection{ORGANIZATION AND RESPONSIBILITIES}

This section discusses the organizations involved in the NDA PDP and the responsibilities of each of these organizations. Figure 1 depicts the organizational flow of communications for the NDA PDP. Other responsibilities of the organizations involved in the NDA Box PDP are provided in the following sections.

\subsection{Carlsbad Field Office}

The CBFO is the review and approval authority for the NDA PDP for boxed DOE TRU waste. Programmatic direction and oversight of the PDP are performed by the Office of the National TRU Program (NTP), the CBFO organization responsible for managing the PDP. The NTP designates a $\mathrm{CBFO}$ employee to be the PDP Appointee.

The CBFO implements the PDP by designating a Program Coordinator (currently the Carlsbad Field Office Technical Assistance Contractor [CTAC]), and by providing program oversight.

The CBFO is responsible for the specification and procurement of NDA PDP standards. An NDA PDP standard is defined as a radioactive source specifically designed, prepared (or acquired), and certified for the NDA PDP. The NDA PDP standards have pedigrees traceable to nationally recognized reference bases such as the National Institute of Standards and Technology. To date, all standards used in the NDA PDP have been manufactured at the Los Alamos National Laboratory. However, sources with appropriate traceability and certification from other programs or commercial sources may be used with CBFO approval. The types of radioactive NDA PDP standards presently in the NDA PDP inventory are listed in section 5.2, Table 2.

The $\mathrm{CBFO}$ is also responsible for the specification and procurement of PDP matrix modules, boxes, and associated components for use in the NDA Box PDP. A PDP matrix box is a modified SWB containing a set of 60 matrix modules that are installed into the box in a specified manner. The installed array of matrix modules contains 24 vertical pass-through holes to allow insertion of PDP standards at known coordinates. Details regarding the design and intended use of the NDA PDP matrix boxes can be found in the specification document associated with each matrix type (INEEL/EXT-02-01271, INEEL/EXT-02-01272, INEEL/EXT-02-01273, INEEL/EXT-02-01274, INEEL/EXT-03-00535). 


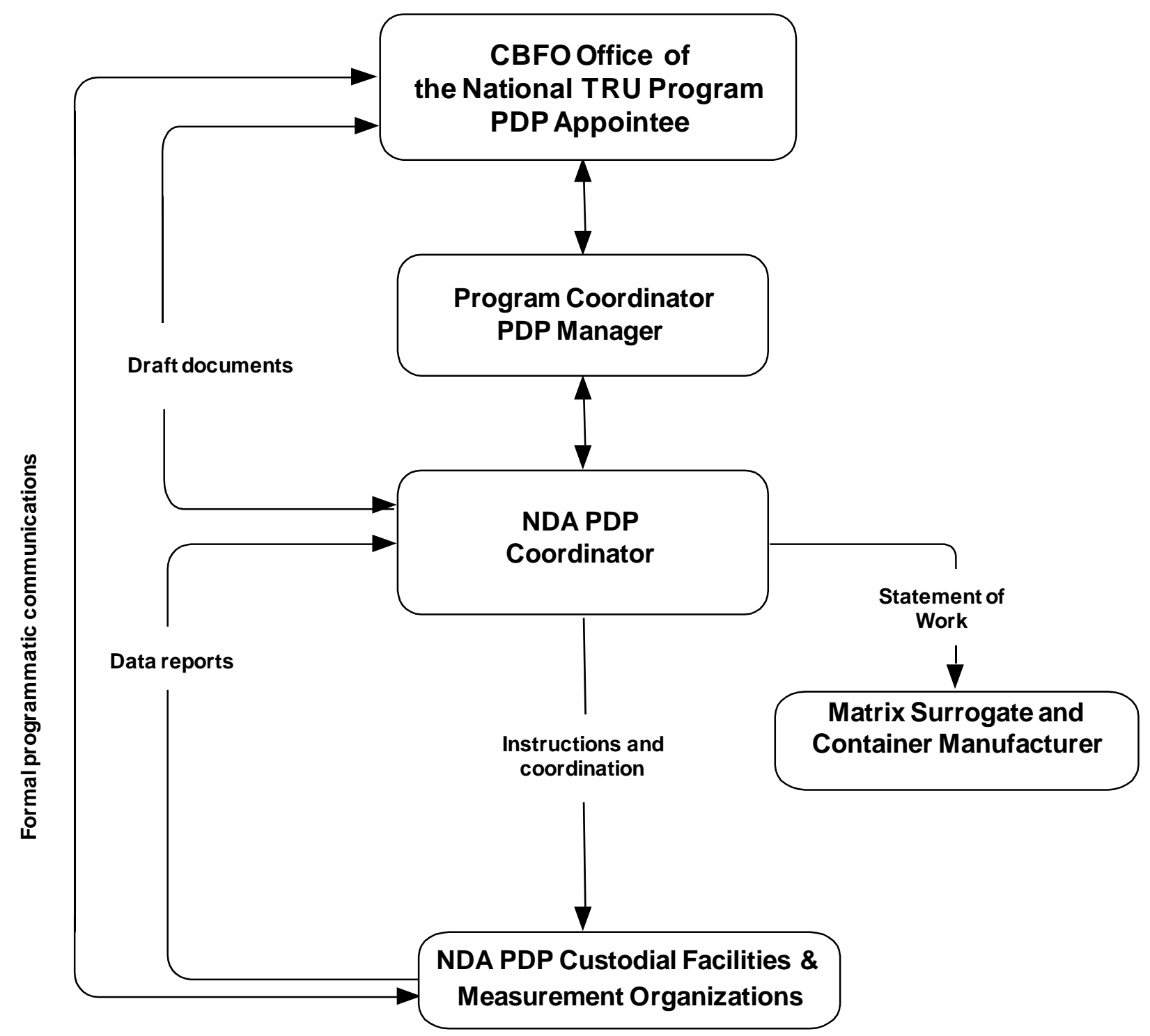

Figure 1. Organization and Communication Flowchart for the NDA PDP 
The NDA PDP is routinely assessed for utility and effectiveness by CBFO through several interrelated activities. These activities include CBFO review of the test results for each NDA PDP cycle; approval of this plan; consideration of the reports and observations of the Program Coordinator, PDP Manager, and NDA PDP Coordinator; feedback from program participants; and comments from other parties such as independent quality assurance assessors. Records of comments, decisions, or proposed actions resulting from the CBFO assessment may take any documented form including, but not limited to, routine program correspondence, meeting minutes, action items, formal review of program documents, assessment reports, and corrective actions.

\subsubsection{PDP Appointee}

The PDP Appointee is the CBFO employee designated for the oversight and individual review and approval authority for the PDP. The CBFO PDP Appointee is responsible for ensuring the implementation of each of the PDP plans by concurring with the designation of the Program Coordinator and by providing technical oversight and coordination for the program.

\subsection{Program Coordinator}

The CTAC is the CBFO-designated organization that functions as the PDP Program Coordinator and technical advisor to the CBFO. The Program Coordinator designates the PDP Manager.

\subsubsection{PDP Manager}

The PDP Manager is responsible for overall management of the PDP and ensures that all PDP responsibilities identified in the PDP Plans and those implemented through the Performance Demonstration Program Management Plan (DOE/CBFO-01-3107) and the applicable CBFO and CTAC management procedures are met. The PDP Manager assigns individuals as coordinators for the three PDP elements: 1) simulated headspace gases, 2) RCRA constituents, and 3) TRU radionuclides using NDA techniques. Each coordinator administers and coordinates their respective PDP functions such as PDP sample component preparation, subcontractor oversight, scheduling, scoring, and generating draft approval status notification memoranda for $\mathrm{CBFO}$ signature and PDP cycle scoring reports.

\subsubsection{NDA PDP Coordinator}

The NDA PDP Coordinator must comply with the requirements and responsibilities stipulated in this plan and the Performance Demonstration Program Management Plan (DOE/CBFO-013107). The NDA PDP Coordinator is responsible for the following activities:

- Distributing and tracking PDP standards and matrix SWBs and components as appropriate to implement the program and to safeguard and secure the PDP materials.

- Maintaining documentation and reconciled inventories on all NDA PDP standards, matrix SWBs, and associated components. 
- Confirming with CBFO and the prospective participants the schedule of a primary NDA PDP cycle approximately two weeks before the planned start date.

- Distributing NDA PDP sample preparation instructions and associated materials to each participating CFAC.

- Ensuring training for the on-site SPT members is in accordance with the NDA PDP Plans.

- Collecting and scoring NDA PDP sample data from the facility measurement organizations.

- Drafting CBFO approval documentation for CBFO authorization of WIPP waste characterization by NDA systems based on their performance in an NDA PDP cycle.

- Drafting CBFO approval documentation of a participating measurement organization's NDA procedures (when the new procedure or revision to an existing procedure potentially impacts waste measurements).

- Preparing cycle-specific scoring reports that provide NDA system-specific and samplespecific scoring results for facility measurement organizations participating in a cycle.

- Ensuring that records of participation and results of all NDA PDP cycles are adequately maintained in a retrievable condition and meet appropriate QA requirements.

- Reviewing changes in the QAPD and WAC that may potentially affect this plan, and providing to $\mathrm{CBFO}$ recommendations for revising the plan as appropriate.

- Assisting in the resolution of disputes related to NDA PDP measurement results, test sample configuration, operational restrictions, and any other issues that may arise during an NDA PDP test cycle.

- Developing technical recommendations to the CBFO regarding conditional NDA characterization approvals.

- Maintaining a current register of participating facility measurement organizations and their measurement system registrations. The registration documentation contains information on each assay system including a unique identity, measurement principles and modes, the NDA PDP test combinations permitted under facility measurement organization/system-specific calibration, and administrative limits.

- Ensuring the NDA PDP test material inventory at each site is sufficient to support cycle implementation.

- Initiating and orchestrating, through the Program Coordinator, the procurement of NDA PDP components to replace lost or damaged parts as necessary.

- Ensuring the responsible parties from each participating facility and other NDA PDP stakeholders are notified in writing when revisions to this plan are available on the National TRU Program Documents area of the WIPP website. 


\subsection{Custodial Facility}

The custodial facility is the physical and organizational entity responsible for NDA PDP activities at the participating DOE site associated with the conduct of the NDA PDP cycles and the storage, care, and proper handling of NDA PDP materials before, during and after cycle activities. It is essential that close contact be maintained between appropriate custodial facility personnel and the NDA PDP Coordinator. The NDA PDP Participating Facility Contacts Form, found in Appendix A, must be provided to the NDA PDP Coordinator by an appropriate custodial facility manager. The form must be updated and forwarded to the NDA PDP Coordinator annually or when contact information changes.

Each custodial facility is required to provide the NDA PDP Coordinator, in writing or by e-mail, with the name, telephone number, and address of a custodial facility assay coordinator (CFAC) to serve as the single contact person responsible for on-site administration and conduct of the NDA PDP (e.g., execution of cycle-specific schedules and instructions). The CFAC must be a full time employee or subcontractor stationed at the facility where the NDA system to be assessed is located. The CFAC may also serve as either the PDP standards custodian or the PDP standards configuration attestant, provided the SPT requirements listed below are met. The CFAC must complete general PDP and NDA Box PDP training as indicated in section 7.0, Training.

The custodial facility is also required to provide contact information (name, telephone number, and address) for a PDP sample components custodian. The address provided must be suitable for freight and package delivery of the NDA PDP components and replacement parts. This information shall be supplied in writing or e-mail every time the contact information changes. The PDP sample components custodian may be part of the SPT if the individual meets the qualifications below, but may remain separate with responsibility for the proper care and storage of non-radioactive NDA PDP components.

A two-person SPT consisting of a PDP standards custodian and a PDP standards configuration attestant shall be assigned by each custodial facility organization. The SPT members must, at a minimum, meet the following requirements:

1. Full-time employee or subcontractor of the facility where NDA PDP materials are stored and maintained.

2. Independent of the measurement group being tested; that is, the individual does not participate in assay measurements, reduction or analysis of the assay data, data validation activities, or data reporting for the NDA PDP samples that he or she has prepared.

3. Trained to applicable site quality assurance (QA) requirements in accordance with the training provided through section 7.0 of this plan.

4. Qualified to handle radioactive materials (PDP standards custodian only). 


\subsubsection{Custodial Facility Assay Coordinator (CFAC)}

The CFAC is responsible for the following NDA PDP cycle activities throughout the year:

- Ensuring timely completion of necessary and requested communication, verbal and written, with the NDA PDP Coordinator regarding all aspects of the conduct of a given NDA PDP cycle.

- Overseeing and managing schedules and PDP sample transfers between the various assay systems at the site.

- Receiving and distributing cycle materials and documents to the SPT in a timely manner.

- Obtaining properly executed SPT documentation from the SPT and submitting it to the NDA PDP Coordinator within two weeks of the return of the final cycle sample to the SPT.

Note: The proper approval of NDA systems cannot be completed without the SPT documents (i.e., properly signed sample configuration forms and sample custody forms) being in the possession of the NDA PDP Coordinator. The completed custody forms and configuration forms are QA records.

- Providing notice and contact information for changes in personnel as soon as possible when changes have occurred.

- Obtaining annual inventory information from the PDP sample components custodian and the PDP standards custodian for submission to the NDA PDP Coordinator each January.

- Disseminating information from revisions to this plan to all affected members of the SPT and affected managers of those members. (Note: The NDA PDP Coordinator will notify the CFAC when revisions to this plan have been posted to the National TRU Program Documents area of the WIPP website.)

- Returning all unused materials provided for a cycle to the NDA PDP Coordinator along with the completed configuration and chain of custody forms as soon as possible and within two weeks following return of the final NDA PDP sample of the cycle.

- Forwarding all relevant communications originating from the SPT to the NDA PDP Coordinator.

\subsubsection{Sample Components Custodian}

The designated PDP sample components custodian has the following NDA PDP responsibilities:

- Ensuring all NDA PDP components are properly stored and controlled to prevent unauthorized use, loss, or damage. Proper storage is defined as indoors, protected from exposure to water, and protected from physical damage (e.g., stacking on top of other SWBs, unnecessary exposure to forklifts). The components must be maintained in the same condition as when they were provided to the participant. The SWBs and 
components must be kept within the DOE free release limits for radioactive contamination.

Note: PDP SWBs are only to be used for NDA PDP purposes unless written permission has been granted by $\mathrm{CBFO}$ for other purposes. Questions concerning permissible use shall be directed to the CBFO PDP Appointee or the NDA PDP Coordinator.

- Providing the NDA PDP Coordinator, in writing or by e-mail, the inventory and location of all PDP materials (standards, SWBs, and parts) annually and as requested.

Note: The NDA PDP Coordinator will request the inventory from the PDP sample components custodian each January. The inventory submitted shall include a description of the condition of the PDP sample components (e.g., matrix modules, security lanyards, matrix spacers, source insert fixtures, and locating pins). Digital images of the NDA PDP sample components can be submitted in addition to condition descriptions.

- Notifying the NDA PDP Coordinator of the need for replacement or repair of any NDA PDP components. If minor repairs to SWBs and related materials are needed, the facility may make those repairs with NDA PDP Coordinator approval. This notification must be in writing, preferably by e-mail.

- Returning the SWB or components in accordance with instructions provided by the NDA PDP Coordinator when requested. Replacement parts will be provided by the NDA PDP Coordinator.

- Ensuring proper palleting and packaging of the PDP SWBs and components prior to shipping (see Appendix B for information on shipping and receiving of NDA PDP SWBs).

\subsubsection{Sample Preparation Team (SPT)}

The SPT is a two-person team consisting of a PDP standards custodian and a PDP standards configuration attestant with the following NDA PDP responsibilities:

- Examining, upon initiation of sample preparation for an NDA PDP cycle, all required PDP sample components (e.g., insert fixtures, locating pins, PDP standards, matrix modules, and matrix spacers) identified on the PDP SWB Matrix Configuration Form and the PDP Standard Configuration Form for SWB (Appendix A) prepared by the NDA PDP Coordinator. The purpose of the pre-load examination is to determine if any components are missing or damaged.

- Notifying the NDA PDP Coordinator of any PDP component loss or damage. If the component is a consumable item (such as a tamper indicating device (TID), form, insert tube, or matrix spacer), determining if a spare component is available from the site inventory. If so, replacing the missing or damaged component with the spare, documenting the replacement as appropriate, and notifying the NDA PDP Coordinator of the replacement. If no spare is available, contacting the NDA PDP Coordinator for a replacement. Notification must be made in writing, preferably by e-mail. The notification may be made directly to the NDA PDP Coordinator or through direct communication to the CFAC, who will then communicate the notification to the NDA PDP Coordinator. 
- Selecting the proper reinforced bottom, configuration SWB for installation of NDA PDP matrix modules, standards, and matrix spacers. The PDP standards configuration attestant verifies that the proper SWB is selected for PDP matrix module and radioactive standard loading.

- Identifying each matrix module that will be used to assemble the SWB matrix array, including void modules, per the sample PDP SWB Matrix Configuration Form (Appendix A). The PDP standards configuration attestant verifies that the proper SWB matrix modules and insert fixture matrix spacers were selected for PDP standard loading. The empty SWB sample is produced by installing NDA PDP standards into a configuration box using the source insert fixtures and internal support structure, with no matrix modules.

- Identifying the correct NDA PDP standards using the applicable PDP Standard Configuration Form for SWB. The form provides the SPT with details for placement/positioning of the PDP standard within the sample.

- Coordinating the movement of the PDP configuration SWB, matrix modules, matrix spacers, NDA PDP standards, and related equipment (such as TIDs) into a designated sample preparation area.

- Assembling the matrix module array of the SWB sample following the instructions of the PDP SWB Matrix Configuration Form.

- Emplacing the PDP standards and independently verifying that the source positioning is correct by initialing the PDP Standard Configuration Form for a SWB. If a PDP sample component is damaged, missing, or misplaced, this information must be reported to the CFAC, who will notify the NDA PDP Coordinator.

- Maintaining the integrity of each prepared NDA PDP sample from the time it is sealed until disassembly. Other than the SPT, no observers are permitted during the PDP sample preparation process without the prior permission of the NDA PDP Coordinator. The SPT and any observers shall not divulge any information regarding the loading and configuration of the PDP matrix SWB test samples until the measurement results are released by the CBFO, or as indicated by the NDA PDP Coordinator.

- Preparing an NDA PDP Sample Custody Form (Appendix A) for transfer of the sample to the facility measurement organization.

- Returning in a free release condition any unused Box PDP materials to storage and securing the NDA PDP standards storage area with a TID. A site-specific security system may be used in place of the supplied TID as long as the prevention of unauthorized access to the unused standards or storage-related documentation can be ensured and documented. The site-specific security system must be approved by CBFO.

- Maintaining all records of PDP sample preparation in strict confidence until CBFO distributes a final report, or as notified by the NDA PDP Coordinator.

- Determining the condition of the TIDs that seal the SWB lids and the copy of the PDP Standard Configuration Form for SWB attached to the SWB sample when the sample is returned from the measurement organization. 
- Disassembling the PDP samples as soon as possible after the return of the samples from the measurement organization.

- Documenting the sample integrity and correct positioning of matrix modules and PDP standards utilizing the NDA Box PDP Sample Disassembly Form (Appendix A) during disassembly of the sample.

- Submitting completed SPT documents and unused cycle materials to the CFAC, allowing time for the CFAC to have documents and materials returned within two weeks of the last NDA PDP sample for the cycle being returned to the SPT from the measurement organization. Submission of these documents is a QA records requirement essential to the successful completion of the cycle.

The PDP standards custodian has the following specific NDA PDP responsibilities as part of the overall SPT responsibilities listed above:

- Loading each insert fixture with NDA PDP standards per the PDP Standard Configuration Form for SWB and inserting each loaded insert fixture into the identified SWB matrix module pass-through hole as specified on the PDP SWB Matrix Configuration Form.

- Sealing with a tamper-indicating security seal the provided, imprinted manila envelope containing a copy of the completed PDP Standard Configuration Form for SWB.

Inserting the manila envelope into the provided see-through plastic envelope and affixing it to the PDP SWB with one of the provided TIDs. Recording the TID number on the Custody Form.

- Transferring the PDP samples and custody forms to the measurement organization by obtaining the signature of the measurement organization assay coordinator (MOAC) designated for each prepared PDP sample.

- $\quad$ Signing the appropriate NDA Box PDP Sample Custody Form for each PDP sample returned from the measurement facility after measurement completion.

- Determining the condition of the TIDs on the SWB lids (all components should meet DOE free release standards).

- Breaking the TIDs (if intact) and removing the SWB lids, allowing the insert fixtures containing the PDP standards to be removed.

- Securing the evidence and noting the condition on the custody form if any TID, custody seal, matrix SWB, or standard shows evidence of tampering. Notifying the NDA PDP Coordinator (through or in conjunction with the CFAC) regarding this condition, in writing, preferably by e-mail. The NDA PDP Coordinator, in consultation with CBFO, will provide written instruction as to how to proceed with a test SWB that shows evidence of tampering.

- Implementing or participating in corrective actions as directed by the NDA PDP Coordinator or CFAC.

- Removing each PDP standard from the source insert fixture during disassembly. 
- Documenting the sample integrity and verifying correct positioning of matrix modules and PDP standards utilizing the NDA Box PDP Sample Disassembly Form (Appendix A) during disassembly of the sample.

- Completing the disassembly documentation by signing the NDA Box PDP Sample Disassembly Form and indicating "disassembled" in the comments section at the bottom of the form.

- Coordinating the return of the PDP matrix SWBs with the PDP sample components custodian.

- Returning the PDP standards to the designated, secured storage areas using the facility's storage procedures.

- Ensuring that all completed SPT documentation and unused cycle materials are sent to the CFAC as soon as possible after PDP samples have been disassembled, and ensuring the $\mathrm{CFAC}$ receives the documents and materials in time to meet the two-week deadline for return of the documents and materials to the NDA PDP Coordinator.

- Ensuring that under no circumstances are any modifications performed on the NDA PDP standards.

The PDP standards configuration attestant has the following specific NDA PDP responsibilities as part of the overall SPT responsibilities listed above:

- Ensuring consistent orientation of the SWB for the insertion of matrix modules and for the PDP standard; initialing the PDP Standard Configuration Form to indicate that consistency has been achieved.

- Verifying that positioning of the NDA PDP standards is correct and documenting the verification by initialing the specified space on the NDA PDP Standard Configuration Form for SWB.

- Affixing the appropriate serialized TIDs on the SWB configuration box lids, thereby sealing and securing the test sample for the measurement process.

- Recording the TID number on the NDA PDP Standard Configuration Form for SWB in the space provided.

- Verifying that the source positioning is the same as indicated on the NDA PDP Standard Configuration Form for SWB by completing the NDA Box PDP Sample Disassembly Form during sample disassembly. If not the same, documenting the discrepancy and notifying the CFAC in writing.

- Completing the disassembly documentation by signing the NDA Box PDP Sample Disassembly Form and indicating "disassembled" in the comments section at the bottom of the form. 


\subsection{Measurement Organization}

The measurement organization must provide a point of contact to the NDA PDP Coordinator for all measurement and reporting activities of an NDA PDP cycle in which that organization is involved. The measurement organization's point of contact is the measurement organization assay coordinator (MOAC).

\subsubsection{Measurement Organization Assay Coordinator (MOAC)}

The MOAC is the point of contact for the NDA PDP Coordinator for all measurement and reporting activities of an NDA PDP cycle. The MOAC or designee has the following NDA PDP responsibilities:

- Completing initial, or updating any existing PDP registration forms for each NDA system to be tested in an upcoming NDA PDP cycle and sending the form(s) to the NDA PDP Coordinator. The form will include the system name, calibration range, operational limits, and other administrative information affecting NDA PDP testing. Appendix C contains the registration form and instructions for registering a system for the NDA PDP.

- Responding in writing to written requests from the NDA PDP Coordinator regarding systems that will be participating in upcoming NDA PDP cycles.

- Accepting PDP samples, ensuring adherence to chain-of-custody protocols, and confirming the accuracy of each sample custody form during transfer of the PDP samples from the SPT of the custodial facility.

- Inspecting the condition of the sample seals by checking the TIDs and ensuring they are intact. If a problem exists with the integrity of a TID, the MOAC or designee shall not accept the sample from the SPT member.

- Reviewing, signing, and dating the sample custody form provided that the TIDs are intact and the sample custody forms are properly completed. This custodial signature means that the measurement organization accepts the PDP sample for analysis.

- Documenting on the PDP sample custody form accompanying the PDP sample all subsequent transfers of the PDP sample within the measurement group and the return of the sample(s) to the SPT.

- Ensuring that the final signature on the custody form is made by the SPT when the PDP sample is accepted by the SPT from the measurement organization for disassembly at the conclusion of assay system measurements.

- Ensuring that the facility measurement organization performs six (6) qualified measurements of each PDP sample using the assay procedures that are implemented for routine waste assays, and approved for use in the WIPP waste characterization program. These procedures must have been reviewed and approved by CBFO.

Note: A qualified measurement is one that would be determined by the NDA operator to meet the specifications of the approved counting procedure and would thereby be 
accepted for review by the expert analyst as if the measurement were a single measurement obtained on a WIPP-bound waste sample. It is not permissible to select six (6) qualified measurements from a larger set of qualified measurements and submit them as the NDA PDP cycle measurement replicate set. If, during review of results by the EA or other data reviewing personnel, a measurement or other data problem is determined, one of the following must occur: 1) A measurement organization procedure, which would also be followed for a similar waste sample data problem, is implemented to remedy the identified PDP data problem, or 2) the MOAC or designee notifies the NDA PDP Coordinator about the problem for instruction on how to proceed.

Use of a secondary or additional measurement mode for obtaining isotopic or other necessary data as prescribed by approved procedure is considered to be a part of the overall measurement. Thus, any measurement of this type must follow the same requirements noted above.

- Ensuring the PDP sample is completely removed and replaced between each sequential measurement.

- Completing and reporting analyses as soon as possible, except in cases where one or more of the six (6) measurements are judged by the NDA analyst to be non-compliant as a WIPP waste qualified measurement.

- Ensuring equipment is returned in a clean condition that meets DOE free release standards.

- Identifying instrument failure or operator error based on the methods used by the measurement organization in identifying these issues when analyzing WIPP samples.

Note: If a measurement is determined to be inadequate, contact the NDA PDP Coordinator. It is not acceptable to evaluate for measurement failure by reviewing NDA PDP data and determining the existence of precision problems. If instrument failure or operator error can be identified in accordance with approved procedures, the NDA PDP Coordinator is to be notified. Based on circumstances, the NDA PDP Coordinator may give permission to repeat one or more of the six-replicate measurements in the set prior to submitting data on the Nondestructive Assay Performance Demonstration Program Report Form (Appendix A).

- Ensuring that a signed measurement report for each replicate analysis of each PDP sample is forwarded directly to the NDA PDP Coordinator. The Nondestructive Assay Performance Demonstration Program Report Form (Appendix A) shall be used to report the data to the NDA PDP Coordinator. Continuation sheets may be used if facility measurement organization comments exceed the allotted space. Completed forms shall be signed by a facility measurement organization staff member, generally the Expert Analyst. Reports should contain any other sample measurement information deemed relevant by the facility measurement organization. Corrections to data, or data inadvertently omitted from the report form, will be accepted, with adequate justification, if the NDA PDP Coordinator is notified before the CBFO approval status notification memorandum (see section 4.2) is issued. 
- Ensuring that all analytical reports are received by the NDA PDP Coordinator within 28 calendar days after the cycle start date or a request for extension has been submitted prior to the due date. The start date is provided in the cycle initiation letter sent to the CFAC by the NDA PDP Coordinator. The NDA PDP Coordinator will send a courtesy copy of the cycle initiation letter to the MOAC at the same time the letter is sent to the CFAC.

- Completing and reporting analyses as soon as possible following all reviews required by routine operating procedures. Should these reviews indicate the need for data manipulation commonly allowed by the routine operating procedure that could result in a delay in reporting the results of the analyses, the MOAC should notify the NDA PDP Coordinator of the cause and duration of any delay. The notification must be followed by a written request for extension of the due date with a proposed, revised due date. If granted by CBFO, the extension approval will designate a new due date.

Note: If an extension has not been requested prior to the applicable due date (i.e., either the original due date or any subsequently granted extended due date), CBFO may elect not to receive subsequent data for that NDA PDP cycle depending upon the circumstances (for example, if more than 12 months have passed, plus the one-month grace period, the data cannot be certified).

- Ensuring that each NDA PDP report form contains, at a minimum, the following information for each determination:

- the reporting facility measurement organization

- system ID designated during the NDA PDP registration process

- PDP cycle for which the data are being reported

- SWB serial number

- NDA PDP Sample ID from the PDP Sample Custody Form for Nondestructive Assay

- measurement system and method (including software/hardware configuration version) used for each isotope (measurement systems using constant isotope ratios are to be indicated on the report form)

- NDA PDP sample measurement replicate number corresponding to the analytical data

- activity in curies for each isotope identified and quantified from the list in section 5.2

- total uncertainty for each identified isotope at one standard deviation

- total ${ }^{239} \mathrm{Pu}$ fissile gram equivalent (g) and associated total uncertainty at one standard deviation

- total TRU alpha activity and associated total uncertainty (curies) at one standard deviation

- thermal power and associated uncertainty at one standard deviation (W)

- elapsed counting time

- date and time of sample analysis

- the reason for no value when not reported (e.g., $<$ minimum detectable concentration $(\mathrm{MDC}))$ 
- Maintaining in a traceable and auditable condition all records generated by the measurement organization during the conduct of an NDA PDP cycle. All such records are QA records that must be maintained in storage conditions and for durations as required by the QAPD and other implementing QA documents and procedures.

- Reviewing the draft CBFO approval status notification memorandum (ASNM) (see section 4.2) provided by the NDA PDP Coordinator. This review will occur prior to the NDA PDP Coordinator submitting the ASNM to CBFO for final approval, signature and subsequent transmittal to the stakeholders. This review is to assist in ensuring that correct information is contained within the memorandum. The MOAC will:

- ensure the proper identity of the system, procedures, and other relevant information provided to the NDA PDP Coordinator by the measurement organization during the cycle are referenced in the ASNM.

- request the NDA PDP Coordinator clarify the language used in the memorandum, if necessary.

- request the NDA Coordinator to seek final resolution by CBFO regarding any disapproval(s) or conditional approval(s) if the approval status or conditions imposed are disputed, with technical justification, by the measurement organization.

\subsection{EVALUATION OF PERFORMANCE DATA}

\subsection{General Criteria}

In the NDA PDP, measurement system performance is evaluated using the measurement data precision and bias. Precision is defined as the percent relative standard deviation (\%RSD); the standard deviation of the six sequential replicate measurements divided by the known value times $100 \%$. Bias is the systematic error component of the total measurement uncertainty determined as the ratio of the mean of the measurement replicate set to the known value times $100 \%$, or the percent recovery $(\% \mathrm{R})$.

The precision acceptance criteria vary as a function of the activity range of the sample and whether the NDA PDP sample matrix is considered to be interfering or non-interfering. The bias acceptance range does not vary as a function of sample alpha $(\alpha)$ activity, but does vary as a function of sample matrix category (interfering or non-interfering). Categorized $\alpha$ activity ranges that apply to the NDA PDP data acceptance criteria are listed in Table 1. See Appendix D for an explanation of the origins and statistical basis for the precision and bias acceptance criteria. 
Table 1. NDA PDP activity ranges and associated scoring acceptance criteria.

\begin{tabular}{|c|c|c|c|c|c|}
\hline & \multicolumn{2}{|c|}{$\begin{array}{c}\text { Maximum Measured } \\
\text { Precision }^{\mathrm{b}}\end{array}$} & \multicolumn{2}{|c|}{$\begin{array}{c}\text { Bias Range } \\
\left(\% \mathrm{R}_{\mathrm{L}} \text { and } \% \mathrm{R}_{\mathrm{U}}\right)\end{array}$} \\
\hline $\begin{array}{c}\text { Activity } \\
\text { range }\end{array}$ & $\begin{array}{c}\text { Range of sample } \\
\text { activity in } \\
\alpha \text {-curies }\end{array}$ & $\begin{array}{c}\text { Non- } \\
\text { interfering } \\
\text { matrix } \\
\text { (\%RSD) }\end{array}$ & $\begin{array}{c}\text { Interfering } \\
\text { matrix } \\
\text { (\%RSD) }\end{array}$ & $\begin{array}{c}\text { Non- } \\
\text { interfering } \\
\text { matrix } \\
(\% \mathrm{R})\end{array}$ & $\begin{array}{c}\text { Interfering } \\
\text { matrix } \\
(\% \mathrm{R})\end{array}$ \\
\hline Low & $>0$ to 0.02 & 14 & 16 & $\begin{array}{c}\text { Lower: } 70 \\
\text { Upper: } 130\end{array}$ & $\begin{array}{c}\text { Lower: } 40 \\
\text { Upper: } 160\end{array}$ \\
\hline Mid-Low & $>0.02$ to 0.2 & 10.5 & 12 & $\begin{array}{c}\text { Lower: } 70 \\
\text { Upper: } 130\end{array}$ & $\begin{array}{c}\text { Lower: } 40 \\
\text { Upper: } 160\end{array}$ \\
\hline $\begin{array}{c}\text { Mid- } \\
\text { High }\end{array}$ & $>0.2$ to 2.0 & 7 & 12 & $\begin{array}{c}\text { Lower: } 70 \\
\text { Upper: } 130\end{array}$ & $\begin{array}{c}\text { Lower: } 40 \\
\text { Upper: } 160\end{array}$ \\
\hline High & $>2.0$ & 3.5 & 6 & $\begin{array}{c}\text { Lower: } 70 \\
\text { Upper: } 130\end{array}$ & $\begin{array}{c}\text { Lower: } 40 \\
\text { Upper: } 160\end{array}$ \\
\hline
\end{tabular}

$\% \mathrm{R}=$ percent recovery

$\% \mathrm{RSD}=$ percent relative standard deviation

a. Applicable range of TRU activity contained in a PDP sample; units are curies of alpha-emitting TRU isotopes with half-lives greater than 20 years.

b. Measured precision that must be met to satisfy the precision criteria at the $95 \%$ upper confidence bound, based on six replicates. The values are one relative standard deviation referenced to the known value for the test.

c. $\quad \% R_{L}$ and $\% R_{U}$ values used in Equation 3 to determine the $95 \%$ confidence bound for the ratio of the mean of the measured values to the known value, expressed as a percent.

\subsection{Scoring the Test Data Precision}

Purpose: To demonstrate compliance with the NDA PDP data acceptance criteria for precision, NDA measurement results from six replicate analyses of an NDA PDP sample of known total TRU $\alpha$-activity and known matrix category (i.e., interfering or non-interfering) are used to determine measurement system precision.

Criteria: The results reported for total TRU $\alpha$-activity from the six replicate measurements of an NDA PDP sample shall not exceed the allowable \%RSD of Table 1, column 3 for the noninterfering matrix samples, or column 4 for the interfering matrix samples.

Method: The analytical results from the six replicate measurements of an NDA PDP sample are used to calculate the \%RSD:

$$
\% \mathrm{RSD}=100 \% \times \frac{1}{\mu_{0}} \sqrt{\frac{1}{n-1} \sum_{i=1}^{n}\left(x_{i}-\bar{x}\right)^{2}}
$$


where:

$$
\begin{aligned}
& x_{i}=\text { replicate sample value } \\
& n=\text { number of replicate measurements } \\
& \begin{aligned}
\mu_{0} & =\text { actual known PDP sample value } \\
\bar{x} & =\text { replicate sample mean, defined by } \\
\qquad \bar{x} & =\frac{1}{n} \sum_{i=1}^{n} x_{i}
\end{aligned}
\end{aligned}
$$

The measured \%RSD is compared with the applicable limits listed in Table 1 . If the \%RSD value determined from the measurement data is less than or equal to the matrix-type and total TRU $\alpha$-activity range-specific value in Table 1 , column 3 or 4 , the measurement system passes the precision test for that sample.

Actions: Any sample for which results exceed the appropriate acceptance criterion for precision will be scored as failed. The impact of failing an acceptance criterion is given in section 4.2. In accordance with section 4.2, the measurement organization assay coordinator is responsible for ensuring that appropriate corrective actions are taken, if deemed necessary by the measurement organization or, subsequently, by CBFO.

\subsection{Scoring the Test Data Bias}

Purpose: NDA results for replicate analyses for NDA PDP samples of known TRU $\alpha$-activity and known matrix category (i.e., interfering or non-interfering) are used to determine the bias associated with measurement system determinations of total TRU $\alpha$-activity. In the NDA PDP, the total bias determined using the six replicate measurement results includes components attributable to both variance and bias, including effects due to sample matrix configuration and nuclear material characteristics.

Criteria: The results reported for total TRU $\alpha$-activity must make true Equation 3 . The selection of the appropriate criteria $\left(\% R_{L, U}\right.$ values) specified in Table 1 is based on the matrix category (i.e., interfering or non-interfering) but does not vary with activity level.

Method: Measurement system bias shall be evaluated by calculating the sample mean of the six sequential replicate measurements of the NDA PDP sample.

For evaluation of sample-specific measurement system bias limits, the Student's $t$-distribution $t_{0.975}$ percentile value is used in conjunction with the sample mean ( $\bar{x}$ as defined in equation 2 ), the percent relative standard deviation (\%RSD of the sample replicates as defined in Equation 1) and the appropriate lower and upper bounds $\left(\% \mathrm{R}_{\mathrm{L}}\right.$ and $\left.\% \mathrm{R}_{\mathrm{U}}\right)$ in columns 5 and 6 of Table 1 (as determined by matrix type). The equation that establishes the bias acceptance criteria is expressed as: 


$$
\left(\% \mathrm{R}_{\mathrm{L}}+1.049 \times \% \mathrm{RSD}\right)<\left(100 \% \times \frac{\bar{x}}{\mu_{0}}\right)<\left(\% \mathrm{R}_{\mathrm{U}}-1.049 \times \% \mathrm{RSD}\right)
$$

where:

$$
\begin{aligned}
\% \mathrm{R}_{\mathrm{L}} & =\text { low percent recovery limit specified in Table } 1, \text { column } 5 \text { or } 6, \text { as appropriate } \\
\% \mathrm{R}_{\mathrm{U}} & =\text { upper percent recovery limit specified in Table } 1, \text { column } 5 \text { or } 6, \text { as appropriate } \\
\bar{x} & =\text { replicate set average (sample mean), defined in Equation } 2 \\
\mu_{0} & =\text { actual known PDP sample value }
\end{aligned}
$$

The measurement will pass the bias acceptance criterion if Equation 3 is satisfied and will fail if Equation 3 is not satisfied.

Actions: If any measurement system produces results that do not satisfy the appropriate acceptance criterion for bias, then that NDA system will be scored as failed for that sample. The impact of failing an acceptance criterion is given in section 6.3. In accordance with section 4.2, the measurement organization assay coordinator is responsible for ensuring that appropriate corrective actions are taken, if deemed necessary, initially by the measurement organization or subsequently by $\mathrm{CBFO}$.

\subsection{WIPP QUALIFICATION BASED ON NDA PDP TEST PERFORMANCE}

\subsection{General Considerations}

The primary purpose of the NDA PDP is to independently acquire data from participating NDA measurement systems for the evaluation of the system and facility measurement organizations' capabilities to produce data that meet the quality requirements for the WIPP, and to reveal any technical or quality assurance-related deficiencies that may negatively impact the characterization of WIPP wastes. Through NDA PDP evaluations, information is gathered on: system performance, NDA measurement organization management quality processes, NDA procedural adequacy, effectiveness and implementation, corrective action effectiveness; and overall data quality assurance. Finally, the NDA PDP functions to provide technical justification to $\mathrm{CBFO}$ for approving (conditionally or without condition) or disapproving NDA systems, procedures, and organizations for making qualified WIPP waste NDA measurements.

Where possible, the procedures and measurement system parameters used to analyze NDA PDP samples are to be the same as those used in the analysis of WIPP wastes. Due to the fact that NDA PDP samples are analyzed six separate times (unlike WIPP waste samples), the procedure for conducting these replicates will be unique to the NDA PDP. Because count time determinations for NDA PDP samples are directly related to the precision of the results and are scored against NDA PDP criteria, the NDA PDP sample count times must have a direct, procedural correlation with count time determinations for WIPP waste samples. Acceptable Knowledge (AK) does exist with respect to NDA PDP samples (e.g., matrix type, nominal density and fill height). Thus, when the PDP sample AK corresponds to actual waste AK, in which count time protocol is specified or formulated, the count time protocol must also be applied to the NDA PDP samples. Any deviations from such protocol must either have a real waste sample procedural justification or specific and documented approval from CBFO. 
The CBFO determines the approval status of a measurement organization's NDA measurement capability for any individual NDA system based on passing or failing NDA PDP scoring criteria. A passing or failing score for a given criterion of the NDA PDP is related to the characteristics of the PDP sample(s) analyzed and is therefore subject to evaluation based upon all of the following specifics of the test: 1) the NDA system;2) the sample matrix; 3) the activity type; 4) the activity range; and 5) the particular scored parameter (bias or precision). Thus, a pass or fail in the NDA PDP has specific, though potentially broad technical and programmatic ramifications.

Obtaining a passing score on all criteria for all samples presented to an NDA system within an NDA PDP cycle provides CBFO with the evidence and justification for not imposing any restrictions upon WIPP measurements made by that NDA system during the approval period. Any restrictions that have not been addressed by the measurement organization through CBFOapproved corrective action, recalibration, etc., continue as restrictions upon the system through the subject cycle approval period. Approval is based upon the use of the measurement organization's CBFO-approved procedures for samples that exhibit characteristics within the approved system's various parameter calibration ranges.

A failure of an NDA system to meet one or more of the scoring criteria may require investigation of the cause of that failure. It is the responsibility of the affected measurement organization, with assistance and oversight by the NDA PDP Coordinator and other CBFO technical support staff, to establish the most likely causes for their failure. Once the cause(s) are reviewed and concurred upon by $\mathrm{CBFO}$, the cause(s) of failure must be evaluated for potential effect upon WIPP waste analyses. It is incumbent upon the participating measurement organization to evaluate and demonstrate, with technical justification, the impact that an NDA PDP criterion failure could have on WIPP waste measurements. CBFO is the final authority on establishing this impact determination.

If the measurement organization can demonstrate that the cause(s) of NDA PDP criteria failure has no impact on routine WIPP waste analyses, no restrictions will be placed upon the system. Based upon the causes of failure, CBFO may determine that multiple systems within the organization should be restricted. Restrictions may include disapproval of all measurements made by the system(s) or may be conditional approval restricting the system(s) from performing measurements for WIPP based on certain waste parameters such as waste types, activity types, activity levels, waste densities, measurement modes within an NDA system, or any other reasonable restriction that is determined through the root cause process as technically justified for prevention of unacceptable data quality for meeting the WIPP waste acceptance criteria.

As specified in section 4.2, CBFO will provide a written ASNM documenting approval, disapproval, or conditional approval. The ASNM will provide the basis for any restrictions. For conditional approvals, the ASNM will specify the restrictions that are imposed by CBFO. The measurement organization is requested to review the draft ASNM for comment and clarification prior to the finalization of the memorandum (see section 2.4.1).

If an NDA system has been conditionally approved or not approved, the measurement organization may choose to take one of the following courses of action: 
- Accept the conditional approval or disapproval with no further action (Note: accepting disapproval will require the measurement organization to halt the use of the system for performing qualified NDA measurements on WIPP waste until the system can be approved or conditionally approved. Likewise, accepting conditional approval limits the system to making qualified WIPP measurements only on samples meeting the specified conditions of the approval);

- Challenge the conditional approval or disapproval with additional data and other technical justifications for a change in the CBFO approval status;

- Initiate a corrective action to eliminate the causes for the condition adverse to quality that resulted in the NDA PDP failure and, if necessary, request a supplemental cycle.

If the measurement organization chooses to initiate corrective actions, the measurement organization must:

1. Identify the basis of the failure that addresses all contributing components, technical and/or administrative, and submit them in a corrective action plan (CAP).

2. Obtain CBFO approval of the CAP.

3. Implement the CBFO-approved CAP and provide evidence that the plan effectively addresses the deficiency and the actions are completed in a corrective action report (CAR).

4. Obtain final CBFO approval, conditional or otherwise, to process WIPP wastes after completion of the CAR.

$\mathrm{CBFO}$ or the measurement organization may require, through the CAP, analysis or additional measurements of NDA PDP test samples as part of the verification that the corrective actions are effective.

If the CBFO determines that the measurement organization is not responsive or is not adequately addressing a condition adverse to quality that has been determined through its participation in the NDA PDP, the CBFO may request a corrective action.

\subsection{Approval Status}

Once the CBFO has determined an organization's measurement systems and methods status as "approved," "conditionally approved," or "not approved," such status shall remain in effect until CBFO is presented justification to change that status. All measurement systems must participate in the PDP cycle annually in order to remain qualified to perform WIPP analyses. Measurement systems obtaining approval status through a supplemental cycle must participate in the next regular primary cycle for reevaluation of their approval status. This participation could change the approval status of a system and will reset the 13-month approval period as stated below.

The approval period for a measurement system begins with the date that signed data reports from an NDA PDP cycle are received by the NDA PDP Coordinator. At the end of the 13th month, a system that has not yet successfully completed the analyses of NDA PDP samples to requalify may choose to proceed at risk with WIPP analyses, or to cease operations. Data obtained at risk cannot be used for certifying waste for shipment to WIPP until: 
- $\quad$ The system and methods used to collect and process the data satisfy the CBFO through successful NDA PDP sample measurement performance, and

- The data obtained during the "at risk" period have been reconciled through the disposition of a nonconformance report.

CBFO approval status notification memoranda are sent to the corresponding DOE Operations Office involved, the participant measurement organization, the U.S. Environmental Protection Agency (EPA) and other relevant stakeholders as designated by CBFO.

\subsection{Scoring Report}

The NDA PDP Coordinator shall review, evaluate, and score the reported data results for all facility measurement organizations and participating NDA systems and compile them into a scoring report. The cycle-specific scoring report is normally scheduled for delivery to CBFO within approximately four weeks after the final data are received from all participants in the cycle, including receipt of revised data as a result of corrective actions. The report shall include the values reported by the measurement facilities, the reference activity values, the acceptance ranges, the pass-fail status of each individual measurement system, and the CBFO approval status of each participating NDA system.

Copies of the scoring report are distributed to each of the DOE Operations Offices involved in the NDA PDP cycle being reported, each of the participating measurement organizations, and other individuals and organizations deemed appropriate by the CBFO. Distribution will be in an electronic format.

\subsection{NDA PDP MATERIAL COMPONENTS}

\subsection{General}

Without written CBFO permission, NDA PDP matrix modules, SWBs, or NTP standards are not to be used for any activity other than the performance of the NDA PDP. Requests for any other uses must be submitted to the $\mathrm{CBFO}$ in written form. The request must specify details of the requested use. If the request is for ongoing usage, a system for accountability must be submitted for CBFO approval. CBFO will provide written approval or disapproval of the request.

Under no circumstances are the NDA PDP standards to be used for measurement system calibration. Under no circumstances are any modifications to be performed on the NDA PDP standards (see section 2.3.3 for custodial facility responsibilities regarding the NDA PDP standards).

The custodial facility is responsible for assigning a secure storage area for all NDA PDP components and meeting applicable safeguards, security, and safety requirements. (See section 2.3.2 for custodial facility responsibilities regarding the non-radioactive NDA Box PDP components. In addition, appendices $\mathrm{B}$ and $\mathrm{F}$ contain details about the non-radioactive NDA Box PDP components.) 


\subsection{NTP Working Reference Material (WRM) Standards of the NDA PDP}

The following primary isotopes are evaluated under the NDA PDP Plans. Of these, the first four are the most significant in terms of WIPP inventory parameters and limits, and are subject to scoring as specified in this plan.
- ${ }^{238} \mathrm{Pu}$
- ${ }^{239} \mathrm{Pu}$
- ${ }^{240} \mathrm{Pu}$
- ${ }^{241} \mathrm{Am}$
- ${ }^{233} \mathrm{U}$
- ${ }^{234} \mathrm{U}$
- ${ }^{238} \mathrm{U}$

National TRU Program PDP standards containing plutonium (powder and granular forms), americium, and depleted and enriched uranium have been specified, fabricated, and distributed to select sites to support the NDA PDP. The uranium isotopes comprise a significant fraction of the DOE inventory and must be accounted for in the repository assessments, but are not subject to NDA PDP scoring. Only transuranic alpha emitting isotopes are considered when scoring NDA PDP data.

Refer to Appendix D for the general specifications of the NTP WRM standards (also referred to as PDP standards) in the current NTP WRM inventory. Detailed information and data concerning the specification, design, fabrication, and traceability for each standard manufactured in each NTP WRM production phase is provided in its respective production plan document (LAUR-96-2277, LAUR-98-213, LA-CP-00-54, LA-CP-00-110, LA-CP-01-208, LA-CP-030072, LA-CP-03-0763). As indicated in Table 2, seven different PDP standard production campaigns were completed, each producing NDA PDP standards with differing nuclear material content.

Table 2. NDA PDP standard inventory.

\begin{tabular}{cll}
\hline & \multicolumn{1}{c}{ Type of Activity } & Production Phase \\
\hline 1. & Weapons-grade plutonium (low mass set) & Phase I \\
2. & Weapons-grade plutonium (high mass set) & Phase II.A \\
3. & Large particle size weapons-grade plutonium & Phase II.B \\
4. & Enriched uranium & Phase II.C \\
5. & Depleted uranium & Phase II.D \\
6. & Increased Am/Pu ratio (relative to the native quantity in & Phase III.A \\
& weapons-grade plutonium) & \\
7. & Heat source plutonium & Phase III.B \\
\hline
\end{tabular}




\subsection{PROCUREMENT}

Procurement or materials necessary for conducting the NDA PDP must comply with the QAPD. In accordance with the QAPD, the responsible purchasing organization maintains all procurement documents and performs all procurement activities.

\subsection{TRAINING}

Each organization involved in the implementation of the NDA Box PDP shall meet the training requirements of the QAPD. Organizations shall retain on file evidence that: 1) personnel have the necessary program documents (controlled or uncontrolled, as applicable) for their use, and 2) personnel have read and understand program-governing documents pertinent to their duties in support of the NDA Box PDP. At a minimum, these documents include applicable portions of the QAPD, the WAC, and this plan.

Training for the NDA PDP CFAC and SPT will be conducted for each of the participating facilities prior to their involvement. The training will be conducted using a CBFO-approved training plan. Successful completion of the training will result in issuance of a certificate of training by the NDA PDP Coordinator that indicates the NDA PDP qualifications of the trained individual. The essential training elements for the CFAC and SPT are as follows:

- Overview of the NDA PDP

- NDA PDP Program Coordination

- NDA PDP Participating Site Responsibilities

- NDA PDP Test Sample Description and Assembly

- NDA PDP Sample Control

- NDA PDP Sample Preparation Responsibilities

- NDA PDP Sample Preparation

- NDA PDP Test Sample Custody

- NDA PDP Test Sample Analysis and Reporting

- NDA PDP Test Sample Return and Disassembly

- NDA PDP Records

Reading material is provided for the initial training through correspondence with the NDA PDP Coordinator. A test is integrated into the training module and must be passed with a proficiency of $90 \%$ or better before proceeding. Upon successful completion of the initial training, the trainee will participate in hands-on cycle activity training at the respective site. This hands-on training is presented by the custodial facility PDP standards custodian or another individual that has successfully completed the training and has performed the function of an SPT member on a previous cycle. Once the hands-on training is successfully accomplished, the certificate is completed and signed by the NDA PDP Coordinator, the hands-on trainer, and the certification recipient. Copies of the training certificate are distributed as QA records. Once the certificate is 
completed and signed by the NDA PDP Coordinator, the individual is qualified to conduct the NDA PDP activities indicated on the certificate.

\subsection{QA RECORDS}

Records generated by the NDA PDP and participating sites during the conduct of a PDP cycle are QA records. All NDA PDP cycle documentation must be maintained in a traceable and auditable condition. Storage conditions and duration must meet the requirements of the QAPD and other implementing QA documents and procedures.

The minimum QA records for the NDA PDP are identified and listed below in accordance with the QAPD requirements. In addition, $\mathrm{CBFO}$ and/or the NDA PDP Coordinator may determine that records of other program activities are QA records and enter them into the QA records system with the same level of control and maintenance.

The QA records may be organized by NDA PDP Plan revision, by NDA PDP cycle, or by other principle, as applicable. These records are nonpermanent records and shall be maintained in accordance with the QAPD requirements. Records disposition, when applicable, will be in accordance with $\mathrm{CBFO} / \mathrm{NTP}$ requirements, approved procedures, and work plans.

All QA records identified in this plan shall be stored in accordance with records storage requirements in the QAPD. Access to QA records will be limited to personnel involved in the program or having related QA or records custodial responsibilities.

The following documents will be maintained as QA records for the NDA PDP:

- $\quad$ PDP plans (all revisions)

- $\quad$ Procurement records

- $\quad$ Radioactive standard and matrix SWB design and production records (each SWB and PDP standard production phase)

- $\quad$ SPT training (training materials, test records, certificates)

- $\quad$ Assay system registration forms

- $\quad$ Records of cycle set-up (notification letters, shipping records, and other correspondence)

- $\quad$ Participant assay reports and supporting forms (assay data report forms, chain-ofcustody records, and configuration forms)

- $\quad$ Scoring reports

- Reviews of corrective actions and supporting data and recommendations made to CBFO

The following matrix of QA records provides a more detailed listing of records and designates responsibility for maintenance. 
NDA PDP Documentation/Records List

\begin{tabular}{|c|c|c|c|}
\hline Document(s) & $\begin{array}{c}\text { QA } \\
\text { Record }\end{array}$ & $\begin{array}{l}\text { Storage } \\
\text { Location }\end{array}$ & Comments \\
\hline 1. PDP Plan revisions & Yes & $\mathrm{CBFO}$ & For each revision. \\
\hline $\begin{array}{l}\text { 2. PDP Plan revisions - comments } \\
\text { and resolutions }\end{array}$ & Yes & $\mathrm{CBFO}$ & For each revision. \\
\hline $\begin{array}{l}\text { 3. } \\
\text { SPT training records } \\
\text { - Tests } \\
\text { - Qualification certificates }\end{array}$ & Yes & CTAC & $\begin{array}{l}\text { NDA PDP Coordinator for each active } \\
\text { SPT member. }\end{array}$ \\
\hline $\begin{array}{l}\text { 4. Approval from CBFO for schedule } \\
\text { and participants }\end{array}$ & Yes & $\mathrm{CBFO}$ & Letter or hard copy of e-mail(s). \\
\hline $\begin{array}{l}\text { 5articipant NDA PDP program } \\
\text { documentation } \\
\text { - Procedures } \\
\text { - Raw data } \\
\text { - Calibration records } \\
\text { - Training records }\end{array}$ & Yes & Participant & $\begin{array}{l}\text { These should be exactly the same as } \\
\text { are maintained/generated in waste } \\
\text { characterization activities; required by } \\
\text { WAC and PDP Plans. }\end{array}$ \\
\hline $\begin{array}{l}\text { 6. SPT training documentation } \\
\text { - Qualification certificates }\end{array}$ & Yes & Participant & $\begin{array}{l}\text { Copies to CTAC and CBFO for SPT } \\
\text { involved in NDA PDP cycle. }\end{array}$ \\
\hline $\begin{array}{l}\text { 7. Correspondence to participants } \\
\text { providing instructions for execution } \\
\text { of an NDA PDP cycle }\end{array}$ & Yes & $\mathrm{CBFO}$ & Copies to CBFO and CTAC. \\
\hline $\begin{array}{l}\text { 8. Sample configuration instructions } \\
\text { accompanying notification }\end{array}$ & Yes & $\mathrm{CBFO}$ & $\begin{array}{l}\text { Developed by the NDA PDP } \\
\text { Coordinator. Confidential until } \\
\text { completion of cycle. }\end{array}$ \\
\hline $\begin{array}{l}\text { 9. Participant correspondence } \\
\text { requesting time extension for } \\
\text { submitting results }\end{array}$ & Yes & $\mathrm{CBFO}$ & $\begin{array}{l}\text { As required by PDP Plans, printed e- } \\
\text { mails to the NDA PDP Coordinator. }\end{array}$ \\
\hline 10. Responses to extension requests & Yes & $\mathrm{CBFO}$ & Responses issued by CBFO. \\
\hline $\begin{array}{l}\text { 11. Participant data packages with cover } \\
\text { letters (including any revisions and } \\
\text { corrections to data) } \\
\text { - Assay data report forms } \\
\text { - Chain-of-custody records } \\
\text { - Configuration forms } \\
\text { - Disassembly forms }\end{array}$ & Yes & $\mathrm{CBFO}$ & $\begin{array}{l}\text { Formally transmitted to CBFO by } \\
\text { NDA PDP Coordinator on completion } \\
\text { of all cycle-specific activities. }\end{array}$ \\
\hline 12. Scoring report & Yes & $\mathrm{CBFO}$ & $\begin{array}{l}\text { Formally transmitted to } \mathrm{CBFO} / \mathrm{CTAC} \\
\text { file copy. CBFO approves distribution } \\
\text { by the NDA PDP Coordinator. }\end{array}$ \\
\hline $\begin{array}{l}\text { 13. Records of QA review of scoring } \\
\text { report }\end{array}$ & Yes & $\mathrm{CBFO}$ & $\begin{array}{l}\text { Formally transmitted to CBFO/CTAC } \\
\text { file copy. }\end{array}$ \\
\hline
\end{tabular}




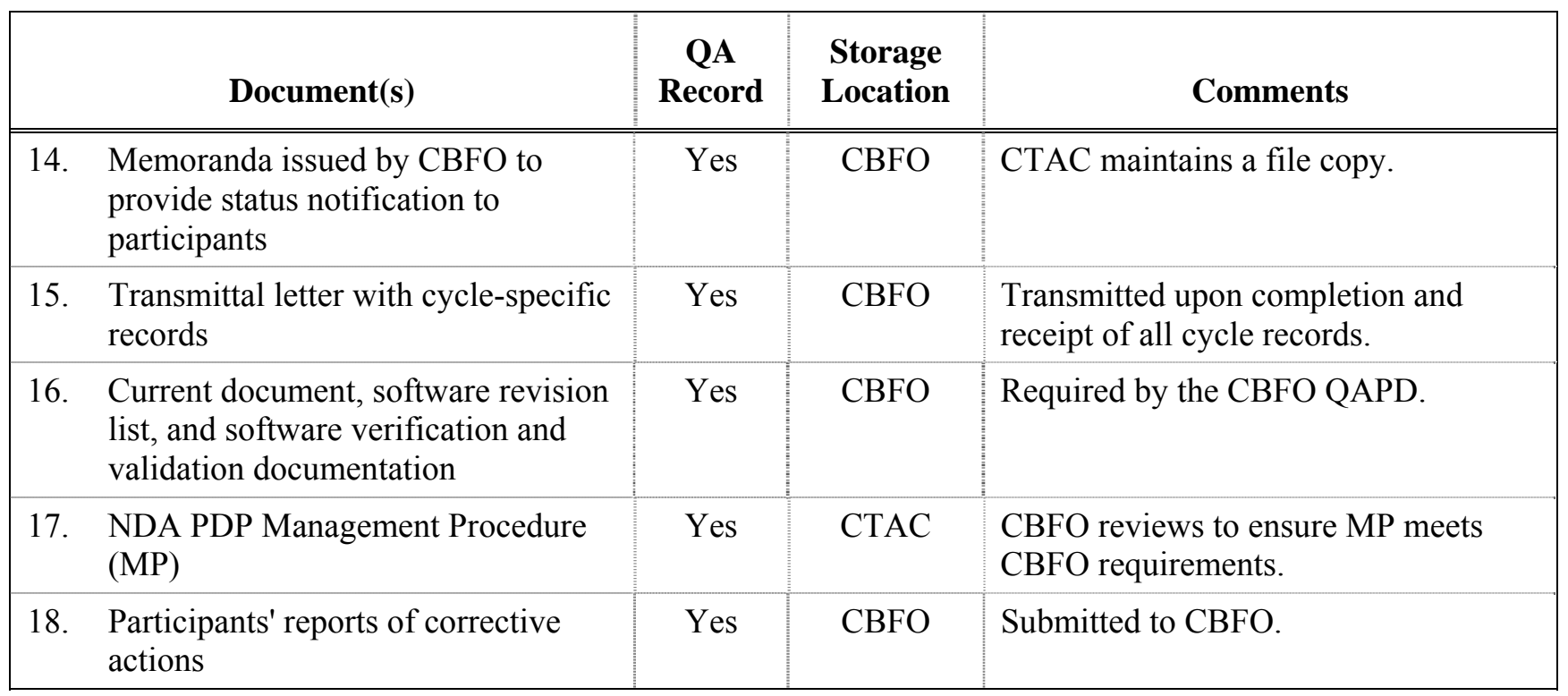

\subsection{GLOSSARY}

ASSAY COORDINATOR - There are two types of assay coordinators functioning in the NDA PDP. One, referred to as the Custodial Facility Assay Coordinator (CFAC) is the facility point-of-contact responsible for receipt of the cycle notification letter, proper sample preparation team (SPT) documentation, and return of the SPT documentation to the NDA PDP Coordinator in a timely manner. A CFAC can be an active participant in the NDA PDP cycle as an SPT member.

The second type of assay coordinator is the Measurement Organization Assay Coordinator (MOAC). This person is generally responsible as the point-of-contact for cycle measurement results, corrective action process, review of draft approval status notification memoranda, and other activities related to the measurement operations of the NDA PDP cycle. It is possible that the CFAC and MOAC roles can be filled by the same individual except that the MOAC cannot participate as an active member of the SPT.

BIAS - The systematic error component of the total uncertainty, that is, a constant positive or negative deviation of the method average from the correct value or an accepted reference value under specific measurement conditions. The percent recovery $(\% \mathrm{R})$ in the NDA PDP summary report includes both systematic (bias) and non-systematic (precision) uncertainty.

CORRECTIVE ACTION - Measures taken to rectify conditions adverse to quality and, where necessary, to preclude their recurrence.

CUSTODIAL FACILITY - The physical and organizational entity responsible for the storage, care, and proper handling of NDA PDP materials during cycle activities and when not in use for NDA PDP activities.

MATRIX SWB - A steel standard waste box (SWB), serial-numbered for the PDP, designed and fabricated with custom reinforcement to contain surrogate waste matrix modules representative of a specified population of the actual DOE waste inventory. Additionally, the matrix SWB has an internal structure for insertion and positioning of PDP radioactive standards. 
MEASUREMENT ORGANIZATION - The entity responsible for the assay of NDA PDP samples and WIPP waste. Frequently this entity is not the same company or entity that functions as the custodial facility or site facility contractor.

MEASUREMENT SYSTEM - The individual NDA instrument that has received a unique NDA PDP registration number. A measurement system may function with various independent modes of assay.

NDA PDP COORDINATOR - An individual responsible for coordinating the technical operations of the NDA PDP, including designation of NDA PDP cycle-specific sample component configuration, procurement of materials and services required for replacement and/or manufacture of new NDA PDP sample components (i.e., NDA PDP matrix SWBs, and consumables), SPT oversight, scheduling NDA PDP cycles, scoring NDA PDP data received from participants, summary report generation, and preparation of other NDA PDP-related CBFO documents as directed by and for approval of the CBFO PDP Appointee.

NDA PDP SAMPLE - A blind sample prepared and sealed by the SPT for subsequent analysis by a measurement system for qualification under the PDP. A PDP sample for the NDA Box PDP is comprised of matrix modules, a configuration SWB and PDP standards installed in accordance with instructions received from the NDA PDP coordinator. Sample matrix and source characteristics will representatively span nominal WIPP waste characteristics expected to be received for characterization by the measurement organization including, but not limited to, isotopics, plutonium concentration, $(\alpha, n)$ reactions, interfering matrices, and source distribution.

NDA PDP STANDARD - A radioactive source specifically designed, prepared, or acquired and certified for the NDA PDP.

NONDESTRUCTIVE ASSAY (NDA) - Assay methods for waste items that do not affect the physical or chemical form of the material. NDA for the PDP includes gamma and neutron techniques only.

NON-INTERFERING MATRIX - Specifies a SWB sample that contains only the supports for the PDP standards (i.e., no installed matrix material), and serves as the non-interfering matrix test sample.

PDP MANAGER - An individual within the Program Coordinator organization responsible for overall performance of the NDA PDP and other elements of the PDP (Headspace Gas and RCRA Constituent Analysis of Solidified Wastes).

PDP STANDARDS CONFIGURATION ATTESTANT - A member of the two-person SPT responsible for verifying the proper configuration of the NDA PDP sample, including emplacement of NDA PDP standards and performing sample security-related procedures.

PDP STANDARDS CUSTODIAN - The lead member of the SPT responsible for coordination of on-site NDA PDP sample preparation activities and handling of PDP standards during sample assembly and disassembly.

PRECISION - A measure of the variance among individual measurements of the same property made under prescribed conditions. Precision is represented in NDA PDP summary reports as a percent relative standard deviation (\%RSD). 
PRIMARY CYCLE - The annual NDA PDP cycle. The primary cycle is included in the Program Coordinator's master project schedules for the NDA PDP and occurs at approximately the same time each year to ensure a site receives the opportunity to gain NDA PDP approval every 12 months.

PROGRAM COORDINATOR - A CBFO-designated organization that administers and coordinates PDP functions. The program coordinator will designate the PDP manager.

SAMPLE PREPARATION PROCEDURE - A procedure generated by the NDA PDP Coordinator for each facility measurement organization for each NDA PDP cycle. This procedure provides instructions to the SPT on configuration of the NDA PDP sample (i.e., emplacement of matrix modules and NDA PDP standards within a specified configuration NDA PDP SWB).

SAMPLE PREPARATION TEAM (SPT) - A two-person team, consisting of an NDA PDP standards custodian and PDP standards configuration attestant that prepare and certify NDA PDP samples for a given facility measurement organization. The SPT is responsible for ensuring that each NDA PDP sample is prepared according to the NDA PDP sample preparation procedures provided by the NDA PDP Coordinator. The SPT ensures proper disassembly and return to storage of all NDA PDP components after analysis by the facility measurement organization. The SPT completes and returns cycle documents as QA records.

SUPPLEMENTAL CYCLE - An NDA PDP cycle in addition to the annual primary NDA PDP cycle. Reasons that a supplemental cycle may be necessary include accommodation of measurement facilities unable to participate in the primary cycle, to support implementation of new or modified systems, or to perform specific testing as directed by the CBFO. Additional supplemental cycles may be conducted on an as-needed basis at CBFO direction.

TOTAL MEASUREMENT UNCERTAINTY (TMU) - The propagated measurement error potential from all bias and precision sources including interference effects such as variable matrices, isotopic compositions, spatial distributions, contaminating radionuclides, and others.

\subsection{REFERENCES}

CBFO-94-1012, Quality Assurance Program Document, current revision, U.S. Department of Energy Carlsbad Field Office, Carlsbad, New Mexico.

DOE/CAO-95-1121, Transuranic Waste Baseline Inventory Report, current revision, U.S. Department of Energy Carlsbad Field Office, Carlsbad, New Mexico.

DOE/WIPP-02-3122, Transuranic Waste Acceptance Criteria for the Waste Isolation Pilot Plant, current revision, Waste Isolation Pilot Plant, U.S. Department of Energy, Carlsbad, New Mexico.

DOE/CBFO-01-1005, Performance Demonstration Program Plan for Nondestructive Assay of Drummed Wastes for the TRU Waste Characterization Program, current revision, U.S. Department of Energy Carlsbad Field Office, Carlsbad, New Mexico. 
DOE/CBFO-01-3107, Performance Demonstration Program Management Plan, current revision, U.S. Department of Energy Carlsbad Field Office, Carlsbad, New Mexico.

INEL-94/0104, Performance Demonstration Program for Nondestructive Assay for the TRU Waste Characterization Program, Initial Cycle Source Design, Idaho National Engineering and Environmental Laboratory, Lockheed Martin Idaho Technologies Company, LLC

LA-CP-00-54, National Transuranic Waste Program Nondestructive Assay Performance Demonstration Program Enriched Uranium Production Plan - Phase II.C, current revision, Los Alamos National Laboratory, Los Alamos, New Mexico.

LA-CP-00-110, National TRU Waste Program NDA PDP Depleted Uranium Production PlanPhase II.D, current revision, Los Alamos National Laboratory, Los Alamos, New Mexico.

LA-CP-01-208, National TRU Waste Program Non-Destructive Assay Performance Demonstration Program Large Particle Working Reference Material Production Plan Phase II.B, current revision, Los Alamos National Laboratory, Los Alamos, New Mexico.

LA-CP-03-0072, National TRU Waste Program Non-Destructive Assay Performance Demonstration Program Increased Americium: Plutonium Working Reference Material Production Plan - Phase III.A, current revision, Los Alamos National Laboratory, Los Alamos, New Mexico.

LA-CP-03-0763, National TRU Waste Program Non-Destructive Assay Performance Demonstration Program Heat Source Plutonium Working Reference Material Production Plan - Phase III.B, current revision, Los Alamos National Laboratory, Los Alamos, New Mexico.

LAUR-96-2277, NDA PDP Working Reference Material Productions Plan - Phase I, current revision, Los Alamos National Laboratory, Los Alamos, New Mexico.

LAUR-98-213, CST-8-PLA-STD-106, NDA PDP High Mass Working Reference Material Production Plan - Phase II.A, current revision, Los Alamos National Laboratory, Los Alamos, New Mexico. 


\section{Appendix A \\ NDA Performance Demonstration Program Forms}


This page intentionally blank 


\section{Appendix A NDA Performance Demonstration Program Forms}

This appendix contains four forms that are essential to the conduct and quality assurance records of the NDA Box PDP cycles. The purpose of each form is described below. Each of the forms is called out in this plan under section 2.0, Responsibilities.

1. The PDP SWB Matrix Configuration Form is the instruction document provided to the sample preparation team (SPT) by the NDA PDP Coordinator for the purpose of assembly and documented disassembly of the test sample matrix module array. This form is initiated by the NDA PDP Coordinator for instruction purposes, but becomes one of the primary QA record documents as it is completed by the SPT during stages of the NDA Box PDP cycle. The documentation on this form provides essential evidence that the proper matrix modules were placed in position as originally designated on the form. Any discrepancies from the original instruction discovered during disassembly must be documented for quality assurance purposes. The completed form is a QA record that must be returned to the NDA PDP Coordinator upon disassembly of the sample as soon after the sample is returned to the SPT as is reasonable.

2. The PDP Standard Configuration Form for SWB is the instruction document provided to the SPT by the NDA PDP Coordinator for the purpose of assembly and documented disassembly test sample PDP standard positioning. This form is initiated by the NDA PDP Coordinator for instruction purposes, but becomes one of the primary QA record documents as it is completed by the SPT during stages of the NDA Box PDP cycle. The documentation on this form provides essential evidence that the proper PDP standards were placed in position as originally designated on the form. Any discrepancies from the original instruction discovered during disassembly must be documented for quality assurance purposes. The completed form is a QA record that must be returned to the NDA PDP Coordinator upon disassembly of the sample as soon after the sample is returned to the SPT as is reasonable.

3. The Nondestructive Assay Box PDP Sample Custody Form is utilized by the SPT and the measurement organization to indicate the time during which each entity is in control of the sample. This document is initiated by the SPT and completed by the SPT upon disassembly. The measurement organization assay coordinator (MOAC) or designee is responsible for the integrity of the NDA PDP samples during the time that the samples are in their possession. The custody form is to be signed and dated by the MOAC or designee for that period of sample possession. The completed form is a QA record that must be returned to the NDA PDP Coordinator upon disassembly of the sample as soon after the sample is returned to the SPT as is reasonable.

4. The NDA Box PDP Sample Disassembly Form must be completed by the SPT. The completed form is a QA record that must be returned to the NDA PDP Coordinator upon disassembly of the sample as soon after the sample is returned to the SPT as is reasonable.

5. The Nondestructive Assay PDP Report Form is used to record the essential information required by the NDA PDP for each measurement of a test sample. This form must be utilized by the MOAC or designee to present the information to the NDA PDP Coordinator once all data review by the measurement organization is complete. One Nondestructive Assay PDP Report Form is required for each NDA Box PDP sample analyzed and for each NDA system used to analyze the sample. The completed forms, signed and dated, are QA records of the NDA PDP cycle.

6. The NDA PDP Participating Facility Contacts Form is provided for convenience, to be utilized by the custodial facility assay coordinator (CFAC) or supervisor thereof, to supply the NDA PDP Coordinator with the names and contact information of those individuals who have responsibility for the conduct and management of the NDA PDP at the WIPP waste packaging and measurement facility. Section 2.0, Responsibilities, describes the responsibilities and expectations of the personnel assigned to the NDA PDP functional titles. In some cases, one individual could function in more than one titled capacity. Though this contact information is essential to the conduct of the NDA PDP, the completed document is not an NDA PDP QA record. 


\section{PDP SWB Matrix Configuration Form}

\begin{tabular}{|c|c|c|}
\hline Facility Name: & \multicolumn{2}{|c|}{ SWB Number ___ of ___ in this Cycle } \\
\hline PDP Sample ID: & \multicolumn{2}{|l|}{ Tier Number: } \\
\hline Matrix Type: & PDP Distribution (Mo/Yr): & Page ___ of \\
\hline
\end{tabular}

Authorized:

NDA PDP Coordinator Date:

(2)

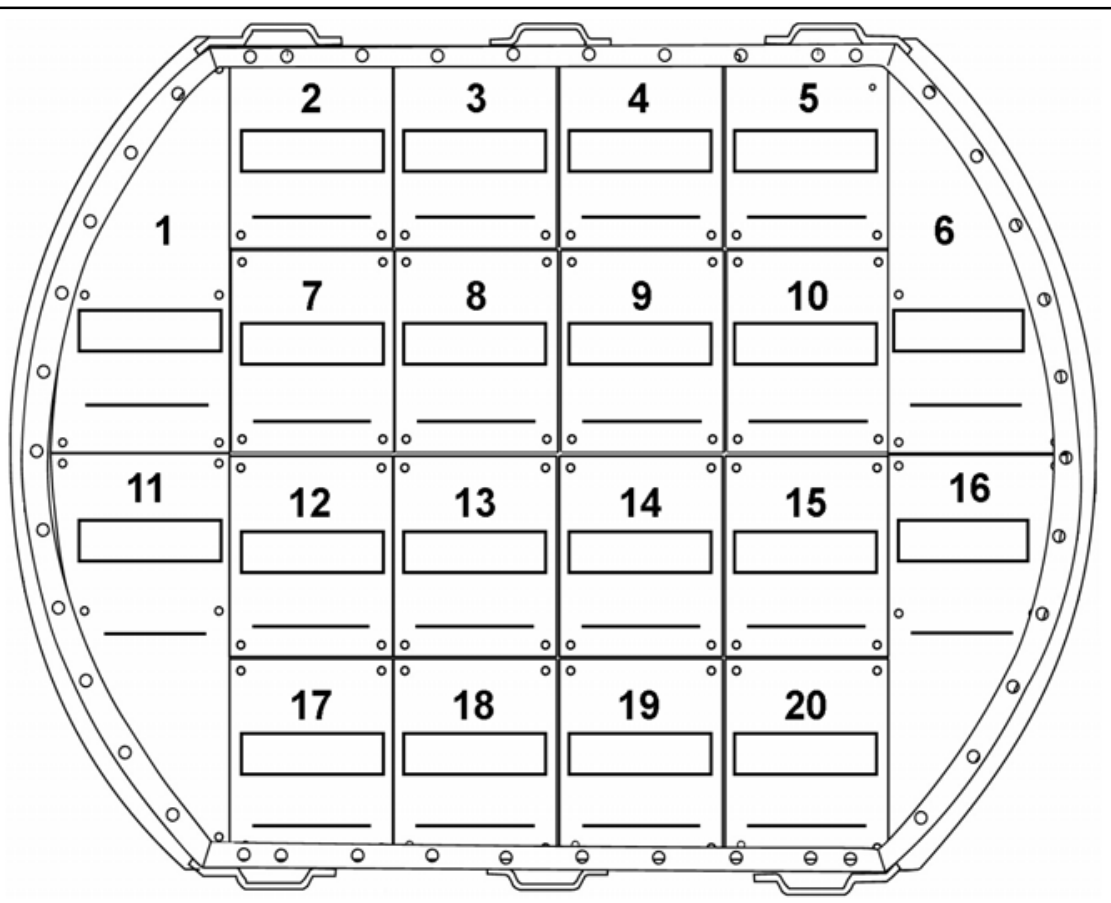


PDP Standard Configuration Form for SWB

\begin{tabular}{|l|l|l|}
\hline Facility Name: & \multicolumn{1}{|l|}{ SWB Number__of__ in this Cycle } \\
\hline PDP Sample ID: & TID Number: & Page _ \\
\hline Matrix Type: & PDP Distribution $(\mathrm{Mo} / \mathrm{Yr}):$ & of \\
\hline
\end{tabular}

Authorized: NDA PDP Coordinator

\begin{tabular}{|c|l|l|l|l|l|l|l|}
\hline \hline $\begin{array}{c}\text { Source } \\
\text { ID }\end{array}$ & $\begin{array}{c}\text { Primary } \\
\text { Nuclear } \\
\text { Materials }\end{array}$ & Activity & Units & $\begin{array}{c}\text { SWB } \\
\text { Position }\end{array}$ & \multicolumn{2}{|c|}{$\begin{array}{c}\text { Rod Position \# } \\
\text { Initial }\end{array}$} \\
\hline & & & & & & \\
\hline & & & & & & \\
\hline & & & & & & \\
\hline & & & & & & \\
\hline & & & & & & \\
\hline & & & & & & \\
\hline & & & & & & \\
\hline
\end{tabular}
(Rod Position \#5)

\begin{tabular}{|l|l|l|l|l|l|l|}
\hline & & & & & & \\
\hline & & & & & & \\
\hline & & & & & & \\
\hline & & & & & & \\
\hline & & & & & & \\
\hline & & & & & & \\
\hline & & & & & & \\
\hline & & & & & & \\
\hline & & & & & & \\
\hline & & & & & & \\
\hline & & & & & & \\
\hline & & & & & & \\
\hline
\end{tabular}

Consistent orientation of the SWB during insertion of the PDP stan dards. Attestant Initials:

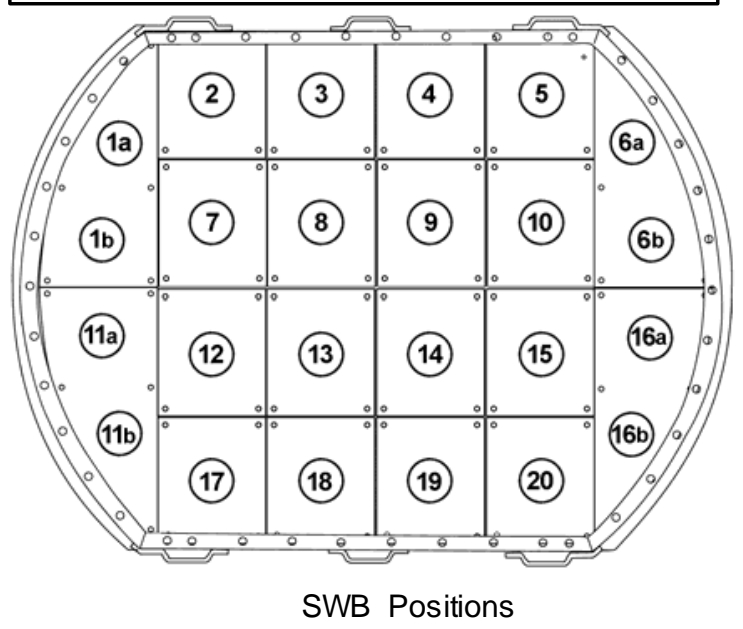

PDP Standards C ustodian: 


\section{Nondestructive Assay Box PDP Sample Custody Form}

SWB Serial Number:

Assay Site:

TID Serial Numbers:

NDA PDP Cycle Number:

Comments:

\section{Sample Preparation}

Sample Preparation Date:

PDP Standards Custodian

Date

$\underline{\text { Initials }}$

Standards properly placed:

Matrix SWB TID properly sealed:

Sample information form attached and sealed:

Standards Configuration Attestant

Date

\begin{tabular}{||l|l|l|l||}
\hline Relinquished by: & Date/Time & Received by: & Date/Time \\
\hline & & & \\
\hline & & & \\
\hline & & & \\
\hline & & & \\
\hline \\
\hline
\end{tabular}

White: NDA PDP coordinator copy on final disposition

Canary: SPT copy on final disposition

Pink: NDA PDP coordinator copy

Gold: SPT copy 


\section{NDA Box PDP Sample Disassembly Form}

SWB Serial Number: TID Serial Numbers:
NDA Box PDP Cycle Number: PDP Sample ID:

Sample Disassembly Record

Sample Disassembly Date:

Condition of Seals and Standards

Configuration Form attached and sealed: $\quad$ Yes $\quad \square \quad$ No

Container TID(s) properly sealed:

Yes $\quad \square \quad$ No

Standards properly placed (Cross out if not applicable):

\begin{tabular}{|c|c|c|c|c|c|c|}
\hline Source & 1 & Yes & $\square$ & No & $\square$ & Condition: \\
\hline Source & 2 & Yes & $\square$ & No & $\square$ & Condition: \\
\hline Source & 3 & Yes & $\square$ & No & $\square$ & Condition: \\
\hline Source & 4 & Yes & $\square$ & No & $\square$ & Condition: \\
\hline Source & 5 & Yes & $\square$ & No & $\square$ & Condition: \\
\hline Source & 6 & Yes & $\square$ & No & $\square$ & Condition: \\
\hline Source & 7 & Yes & $\square$ & No & $\square$ & Condition: \\
\hline Source & $\underline{8}$ & Yes & $\square$ & No & $\square$ & Condition: \\
\hline Source & 9 & Yes & $\square$ & No & $\square$ & Condition: \\
\hline Source & 10 & Yes & $\square$ & No & $\square$ & Conditio \\
\hline
\end{tabular}

Surrogate matrix modules properly placed:

$\begin{array}{lllll}\text { Tier } 1 & \text { Yes } & \square & \text { No } & \square\end{array}$ Condition:

$\begin{array}{lllll}\text { Tier } 2 & \text { Yes } & \square & \text { No } & \square\end{array}$ Condition:

$\begin{array}{lllll}\text { Tier } 3 & \text { Yes } & \square & \text { No } & \square\end{array}$

Comments:

PDP Standards Configuration Attestant

Date

PDP Standards Custodian

Date 


\section{Nondestructive Assay Performance Demonstration Program Report Form}

Assay Facility:

PDP Cycle:

SWB Serial No.:
NDA System ID:

Replicate: of

PDP Sample ID:

Final Result Summary

\begin{tabular}{||l|l|l||}
\hline \multicolumn{1}{|c|}{ Parameter } & Final Result & Total Uncertainty (One Standard Deviation) \\
\hline Total ${ }^{239}$ Pu fissile gram equivalent (g) & & \\
\hline Total TRU alpha activity (curies) & & \\
\hline Thermal Power (W) & & \\
\hline
\end{tabular}

Method Summary

\begin{tabular}{|c|c|c|c|c|c|c|}
\hline & \multirow[b]{2}{*}{ Identification } & \multirow[b]{2}{*}{ Classification } & \multirow{2}{*}{$\begin{array}{l}\text { Associated SOP Identification } \\
\text { (Including Revision No.) }\end{array}$} & \multirow{2}{*}{$\begin{array}{l}\text { Count Time } \\
(\min )\end{array}$} & \multicolumn{2}{|c|}{ Analysis } \\
\hline & & & & & Date & Time \\
\hline \multicolumn{7}{|l|}{ Method 1} \\
\hline \multicolumn{7}{|l|}{ Method 2} \\
\hline Method 3 & & & & & & \\
\hline
\end{tabular}

Individual Isotope Data

\begin{tabular}{|c|c|c|c|c|c|c|c|c|}
\hline \multirow[b]{2}{*}{ Isotope } & \multirow[b]{2}{*}{ Activity Result } & \multicolumn{2}{|c|}{ Uncertainty (1sd) } & \multicolumn{4}{|c|}{ Quantification Method } & \multirow{2}{*}{$\begin{array}{c}\text { Method } \\
\text { Number } \\
\text { (From } \\
\text { Summary) } \\
\end{array}$} \\
\hline & & Count & Total & Direct & Ratio & $\begin{array}{l}\text { Scaling } \\
\text { Isotope }\end{array}$ & $\begin{array}{l}\text { Ratio } \\
\text { Value } \\
\end{array}$ & \\
\hline \multicolumn{9}{|l|}{${ }^{238} \mathrm{Pu}$} \\
\hline \multicolumn{9}{|l|}{${ }^{239} \mathrm{Pu}$} \\
\hline \multicolumn{9}{|l|}{${ }^{240} \mathrm{Pu}$} \\
\hline \multicolumn{9}{|l|}{${ }^{241} \mathrm{Am}$} \\
\hline & & & & & & & & \\
\hline & & & & & & & & \\
\hline & & & & & & & & \\
\hline & & & & & & & & \\
\hline
\end{tabular}

Comments:

Approval: 
NDA PDP Participating Facility Contacts

\begin{tabular}{|c|c|c|c|}
\hline NAME & MAIL ADDRESS & $\begin{array}{c}\text { PHONE/FAX/ } \\
\text { E-MAIL }\end{array}$ & NOTE \\
\hline \multicolumn{4}{|c|}{ Custodial Facility Management Contacts } \\
\hline Operations & & $\begin{array}{l}\text { Voice: } \\
\text { Fax: } \\
\text { E-mail: } \\
\text { Cell: }\end{array}$ & \\
\hline SNM Custodian & & $\begin{array}{l}\text { Cell Voice: } \\
\text { Fax: } \\
\text { E-mail: } \\
\text { Cell: }\end{array}$ & \\
\hline \multicolumn{4}{|c|}{ Custodial Facility NDA PDP Function Contacts } \\
\hline \multicolumn{4}{|c|}{ Assay Coordinator } \\
\hline & & $\begin{array}{l}\text { Cell Voice: } \\
\text { Fax: } \\
\text { E-mail: } \\
\text { Cell: }\end{array}$ & \\
\hline \multicolumn{4}{|c|}{ Components Custodian } \\
\hline & & $\begin{array}{l}\text { Cell Voice: } \\
\text { Fax: } \\
\text { E-mail: } \\
\text { Cell: }\end{array}$ & \\
\hline \multicolumn{4}{|c|}{ PDP Standards Custodian } \\
\hline & & $\begin{array}{l}\text { Cell Voice: } \\
\text { Fax: } \\
\text { E-mail: } \\
\text { Cell: }\end{array}$ & \\
\hline \multicolumn{4}{|c|}{ PDP Standards Configuration Attestant } \\
\hline & & $\begin{array}{l}\text { Cell Voice: } \\
\text { Fax: } \\
\text { E-mail: } \\
\text { Cell: }\end{array}$ & \\
\hline
\end{tabular}


This page intentionally blank 


\section{Appendix B}

\section{Shipment, Receipt, and Use of NDA Box PDP Materials}


This page intentionally blank 


\section{Appendix B Shipment, Receipt, and Use of NDA Box PDP Materials}

This appendix describes the policies and some processes for shipping and receiving NDA PDP materials. This appendix is not meant to be all inclusive of the requirements that will be encountered for movement of NDA PDP materials. In addition to these policies and process guidelines, the shipment and receipt of all NDA PDP materials must meet federal, state, and facility requirements for both the shipping facility and the receiving facility.

\section{B.1 General}

The NDA PDP Coordinator shall coordinate any transfers of NDA PDP materials between sites, and no transfers will occur without written approval from CBFO. The NDA PDP Coordinator will provide the transferring facility with the necessary contact information (names, phone numbers, e-mails, and physical addresses) for the receiving facility. The current custodial facility will notify each receiving site contact prior to the shipping date for NDA PDP materials. The materials will be sent to the address and individual designated by the receiving facility.

For detailed information regarding the receipt, storage, use and shipment of the NDA PDP SWB matrix modules, boxes, and ancillary components, refer to INEEL/EXT-01-00694, National TRU Waste Program Performance Demonstration Program Standard Waste Box Matrix Surrogate, User Manual, May 2001. Contact the NDA PDP Coordinator to obtain a copy of the manual.

The custodial facility assay coordinator at each facility shall notify the NDA PDP Coordinator in writing (e-mail is acceptable) of any changes in contact personnel.

\section{B.2 PDP Surrogate Waste Matrix Modules and SWBs}

The NDA PDP SWB matrix inventory currently consists of seven separate boxes and matrix module sets: two (2) dry combustibles matrix module sets; two (2) mixed metals type matrix module sets; one (1) concrete debris matrix module set; one (1) light metals type matrix module set; and one (1) wet combustibles type matrix module set. Identifying serial numbers are assigned and affixed to each SWB matrix module box. There are two SWB box types, differentiated as: 1) the matrix configuration box, and 2) the matrix module storage box. The configuration box contains an elaborate SWB floor reinforcement structure that provides for the installation and correct positioning of the matrix module array and NDA PDP standard insert fixtures. The configuration box contains additional internal components to secure the matrix/source array in place to prevent configuration shifts during movement and measurement of the SWB PDP sample. The configuration box also has provisions for the attachment of TIDs to ensure the integrity of the sample during the test process. The matrix storage box has none of these added features and cannot be utilized as a configuration box (i.e., PDP sample).

Below is a list of the NDA PDP SWBs by serial number and matrix module type shipped with the specific SWBs. The configuration boxes also serve as storage boxes when the SWB matrix modules shipped with them are not in use as PDP samples.

- Serial\# NDA PDP SWB-01 (configuration box, originally for dry combustibles, "NDA PDP SWB-CMB.XX" series)

- Serial\# NDA PDP SWB-02 (configuration box, originally for dry combustibles, "NDA PDP SWB-CMB.XX" series) 
- Serial\# NDA PDP SWB-03

- Serial\# NDA PDP SWB-04

- Serial\# NDA PDP SWB-05

- Serial\# NDA PDP SWB-06

- Serial\# NDA PDP SWB-07 (storage box, originally for mixed metals, "NDA PDP SWBMET.XX" series)

(storage box, originally for mixed metals, "NDA PDP SWBMET.XX" series)

(configuration box, originally for concrete, "NDA PDP SWBCON.XX" series)

(configuration box, originally for wet combustibles "RFETS SWB-WC.0XX" series)

(storage box, originally for light metals "RFETS SWB480.0XX" series)

Refer to Appendix F for general information on the PDP SWB matrix box design and configuration. Details regarding the design and intended use of the NDA PDP SWBs can be found in the specification documents associated with each matrix type as provided in Appendix F.

The NDA PDP Coordinator shall coordinate any transfers of NDA PDP SWBs and components between sites and no such movement will occur without written approval from CBFO. See Appendix B for additional information on shipment and receipt of NDA PDP materials.

\section{B.3 Receipt of NDA PDP Matrix Modules, SWBs, and Components}

On receipt of NDA PDP matrix SWBs and components, the PDP sample components custodian shall:

- Verify that the serial numbers and physical descriptions of the NDA PDP matrix modules, SWBs, and associated components received match those listed on the shipping manifest.

- Ensure the matrix spacers are included in the shipment as indicated on the manifest. The PDP matrix SWB insert fixtures will need to be removed to assess the presence of the spacers.

- Open any additional shipped packages to verify the content and quantity of items.

- Verify that the matrix modules, SWBs, and ancillary components have not been damaged during shipping. Where shipping manifest discrepancies are noted or damage is found, the assay coordinator and/or the SPT shall secure the items and the assay coordinator and/or the SPT shall notify the NDA PDP Coordinator. If no discrepancy or damage is found, the assay coordinator shall notify the NDA PDP Coordinator in writing or by e-mail, that the shipment was received in acceptable condition.

\section{B.4 Transfer OF NTP Working Reference Material (WRM) Standards (PDP Standards)}

All transfers of NTP WRM standards must have prior written approval from CBFO.

Before shipment of PDP standards, each receiving custodial facility will make appropriate arrangements with the facility's safeguards and radiation safety organizations for storage and accountability. Generally, the designated PDP standards custodian will coordinate with the site safeguards staff to comply with all site special nuclear material (SNM) requirements. All shipping details and arrangements will occur between the trained staff of the DOE facilities of both the shipping and receiving entities. The NDA PDP Coordinator will assist in facilitating the transfer and should remain informed by the shipping and receiving facilities of communications and significant activities associated with the transfer.

At the time of receipt, the PDP custodial facility assay coordinator and/or PDP standards custodian assigned by the custodial facility shall inspect, inventory, and secure the PDP standards. Documentation of these activities must then be provided to the NDA PDP Coordinator. 


\section{Appendix C}

\section{NDA Box PDP System Registration Form}


This page intentionally blank 


\section{Appendix C \\ NDA Box PDP System Registration Form}

This appendix contains the registration form that must be completed by the MOAC or designee, and revised when the information is no longer correct, for an NDA system to be authorized by CBFO to participate in the NDA Box PDP.

\section{General Instructions}

1. Registration forms are to be completed and returned to the NDA PDP Coordinator at least three (3) weeks prior to participation in the PDP.

2. Separate registration is required for each NDA measurement system.

3. After initial submission, the forms need be resubmitted only when a change is made in the registration information.

4. The NDA PDP Coordinator will acknowledge all registration requests and assign a tracking identifier to each registered system.

5. The NDA PDP Coordinator will maintain a current list of all registered systems.

\section{Instructions for Specific Questions}

\section{$\underline{\text { Section A }}$}

1. Enter the full formal name of the measurement system.

2. Enter the acronym by which the system should be referenced.

3. Enter a number associated with this unit (if applicable).

4. Check the appropriate descriptor. "Fixed, Permanent" indicates that the system was intended to be installed permanently at the current location. "Transportable, Nonpermanent" indicates a long-term installation that can be relocated. "Mobile, Trailer" indicates systems intended for routine movement between sites for short-term contracts.

5. Enter the DOE site where the system will be installed for NDA PDP cycle participation.

6. Enter the on-site location designator for the system.

7. Enter the name of the institution/facility/company that owns the system.

8. Enter the name of the institution/facility/company that operates the system.

9. Enter the name of the person who should be contacted for information on the system.

10. Enter the title of the person identified in Box 9.

11. Enter the affiliation of the person identified in Box 9.

12. Enter the mailing address for the person identified in Box 9.

13. Enter the express package delivery address (i.e., street address, not a P.O. box) for the person identified in Box 9.

14. Enter the e-mail address for the person identified in Box 9.

15. Enter the phone number for the person identified in Box 9.

16. Enter the fax number for the person identified in Box 9. 


\section{$\underline{\text { Section B }}$}

1. Enter a description of the system, its principles of operation, and optional modes for assay.

2. Enter the identifier(s) for the written standard operating procedures (SOPs) that are used to operate the system for waste assay.

3. For each measurement mode of the system that may be used, enter the measurement principle (gamma, neutron), mode identification (active neutron, etc.), the procedure documenting selection criteria for selecting the subject modes, and the source of isotopic data used for that mode. Sources of isotopic data may be coded:

$\mathrm{P}=$ isotopic data are measured as an integral part of the primary quantitative assay (e.g., gamma spectrometric methods used for both the quantitative and isotopic data).

$\mathrm{S}=\quad$ isotopic data are derived from a secondary method (e.g., the primary quantitation is by passive neutron assay, but isotopic ratios are obtained from an independent gamma spectrometric method).

$\mathrm{AK}=$ isotopic data are obtained from acceptable knowledge of the waste stream or container.

\section{$\underline{\text { Section C }}$}

1 through 5. Check off Yes or No to each question. For each "Yes," indicate the applicable mode(s) from section B.3.

6. Enter the possible mode(s) from section B.3 for each combination of activity range and waste type. Enter "NA" for combinations for which the system will not be used. If explanatory information is required, enter a number in the comments column and add the number and explanation to section D. For example, a system may have a calibration cut-off that falls at the midpoint of a test range. This may be indicated by accepting the range, but specifying a numerical limit in the comment.

7 through 9. Enter the values and units for any limits on NDA PDP tests that, if exceeded, would prevent the system from assaying an NDA PDP sample.

$\underline{\text { Section D }}$

Add any comments necessary to explain answers in any prior sections or supplemental information useful to the NDA PDP Coordinator in planning effective NDA PDP tests for the system. Attach continuation sheets as needed.

\section{$\underline{\text { Section E }}$}

Enter the requested information for the person submitting the registration form. Sign and forward the original of the form to the NDA PDP Coordinator. 


\section{NDA Box PDP System Registration Form}

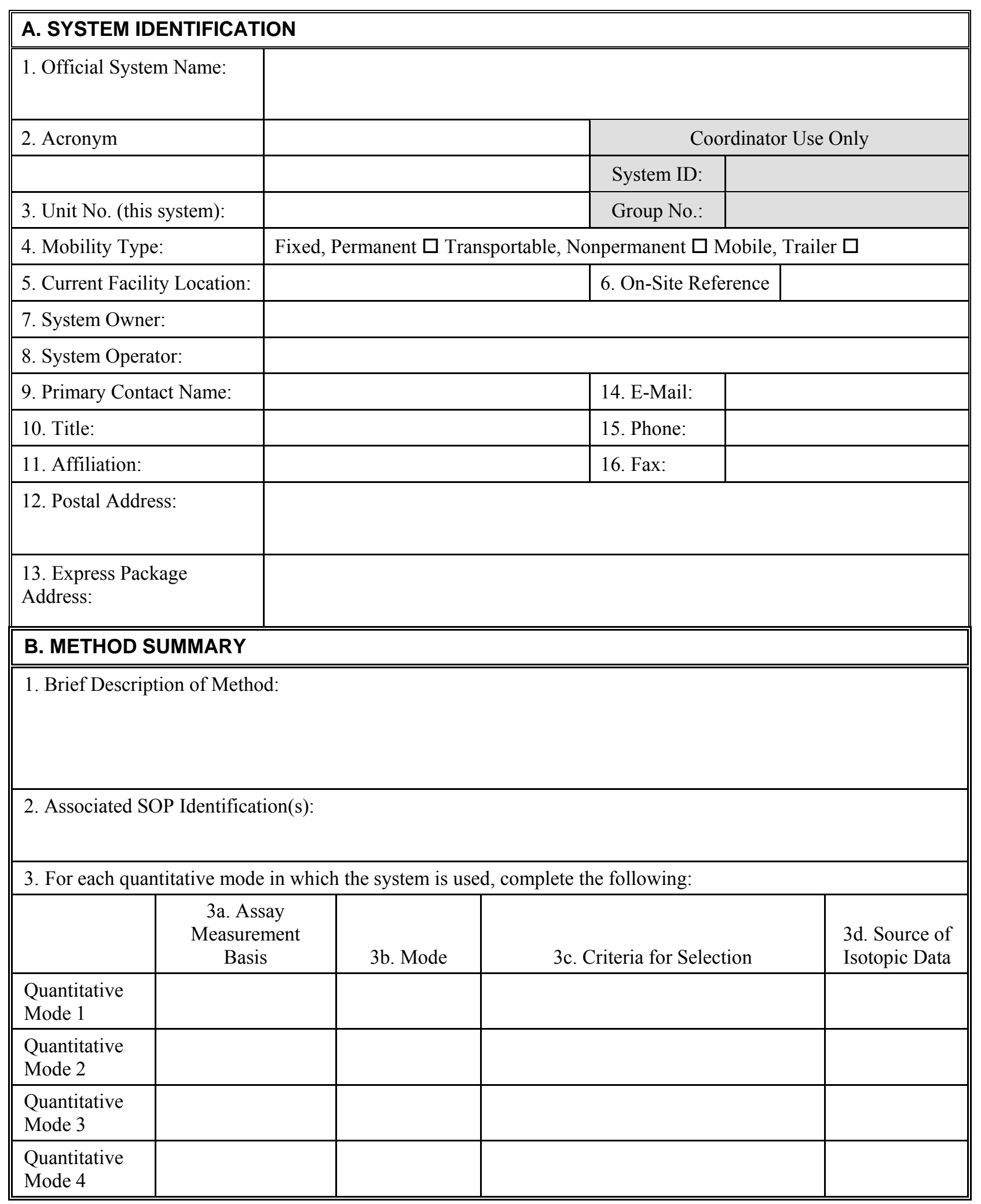




\section{SCOPE AND LIMITATIONS ON SYSTEM USE}

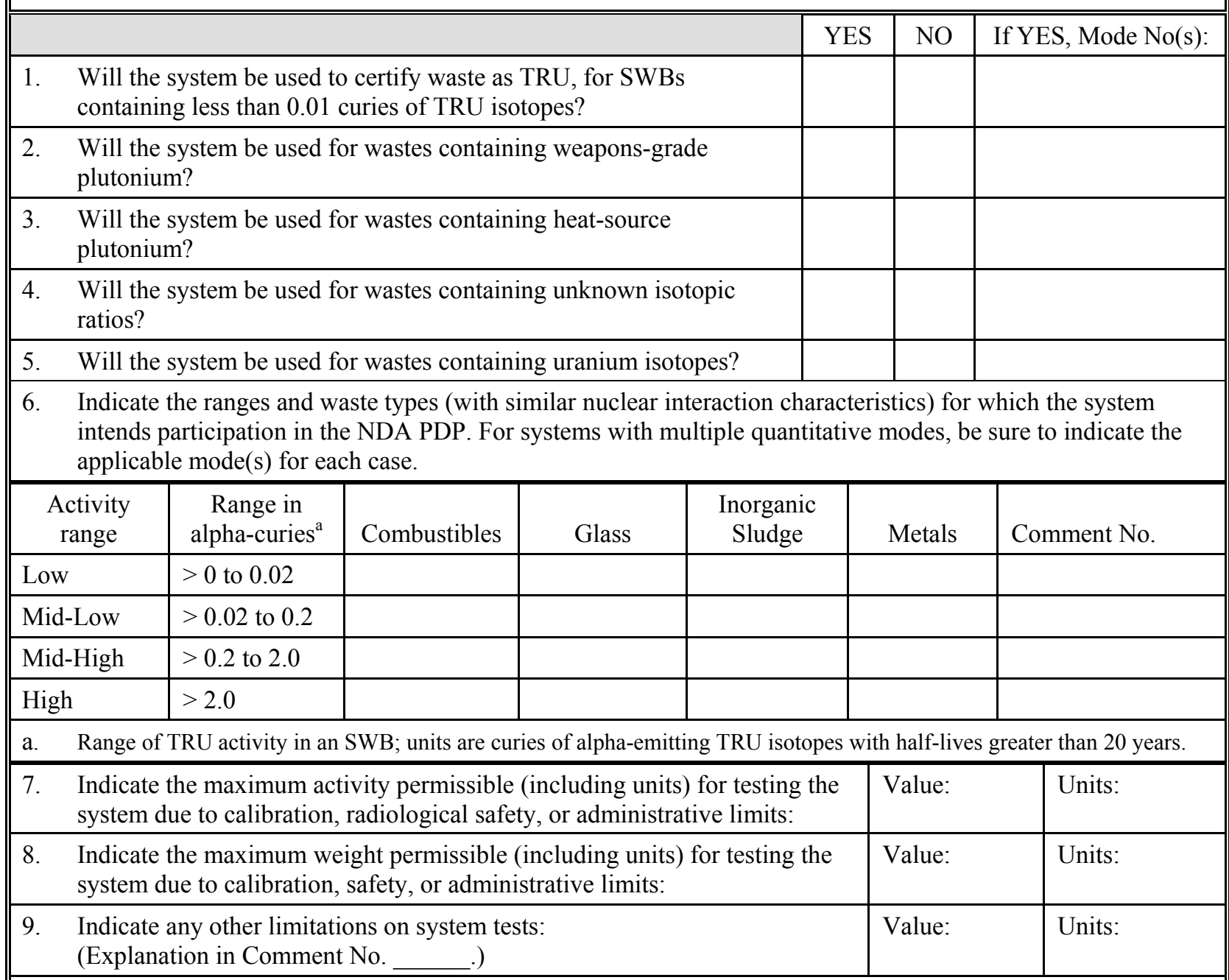

D. COMMENTS (Please add any information relative to participation of this system in the NDA Box PDP)

\section{E. Please register the system described in this application for participation in future cycles of the NDA Box PDP. It is understood that system tracking, test design, approval conditions, participation requirements, and audit follow-up may be based on the information supplied in this application.}

\begin{tabular}{|l|l|l|}
\hline Printed Name: & Signature: & Date: \\
\hline Phone Number: & E-Mail: & \\
\hline
\end{tabular}

F. DISPOSITION (coordinator use only) 


\section{Appendix D}

\section{Statistical Basis of NDA PDP Scoring Criteria}


This page intentionally blank 


\section{Appendix D Statistical Basis of NDA PDP Scoring Criteria}

This appendix provides the basis for the Nondestructive Assay Performance Demonstration Program (NDA PDP) scoring criteria. It specifies how the criteria relate to the Transuranic Waste Acceptance Criteria [WAC] for the Waste Isolation Pilot Plant (DOE/WIPP-02-3122). It further explains the statistical derivation of the limits and bounds criteria of the precision and bias, respectively, for the NDA PDP scoring assessment.

\section{D.1. Definitions}

\section{Limits and Bounds}

This discussion describes two types of bounds or limits: (1) those that define the acceptable precision limits for a NDA measurement system, and (2) the allowable bias range defined by the bounds of the $95 \%$ confidence interval. While the terms "limits" and "bounds" can be used interchangeably, to avoid confusion, the term "limits" is used here only in reference to the PDP precision criteria. Similarly, the term "bounds" is used only to describe the endpoints or bounding values of calculated $95 \%$ confidence intervals for the percent recovery.

\section{Point Estimate}

A point estimate is the best single numerical value that is a good indicator of the underlying parameter of interest. Point estimates contrast with confidence bound estimates, which are interval estimates (since they delineate bounds on confidence intervals). For bias, the point estimate is the mean calculated percent recovery $(\% \mathrm{R})$, relative to the known value. For precision, the point estimate is the percent standard deviation relative to the known value (\%RSD).

\section{D.2. Performance Criteria}

For a non-interfering matrix, the NDA PDP criteria (Table D-1, column 2) specify acceptable limits for the measured precision of an NDA system based on 15 replicate assay measurements. The NDA PDP criteria are derived from the calibration confirmation criteria for NDA systems in the Transuranic Waste Acceptance Criteria [WAC] for the Waste Isolation Pilot Plant (DOE/WIPP-02-3122). The precision criterion, defined as the maximum allowable \%RSD for a non-interfering matrix and given in Table A-3.2 of the WAC, is $20.0 \%$ for 15 replicate assay measurements. This same precision limit of $20 \%$ RSD is given in Table D-1 column 2 for the lowest TRU alpha activity range.

The measured precision, based on 15 replicates, is only an approximation of the true system precision. Implicit in each limit for the measured precision is a corresponding 95\% upper confidence endpoint value on the true system precision. These upper limits are stated explicitly in Table D-1. Precision criteria for NDA PDP tests for six replicate assay measurements, derived in relation to the upper confidence limits for 15 replicate assay measurements, are given in Table D-1 for non-interfering and interfering matrices.

The accuracy criterion for non-interfering matrix bias, as specified in the WAC, section A.3, Calibration Confirmation, is a maximum difference of $\pm 30 \%$ from $100 \%$ recovery. This criterion has been adopted for use in the NDA PDP for the non-interfering matrix bias criterion (see Table D-1). The PDP criteria for bias for the interfering matrices, shown in Table D-1, are less restrictive in consideration of the complexities involved with matrix interferences. 
Table D-1. NDA PDP performance criteria.

\begin{tabular}{|c|c|c|c|c|c|c|}
\hline \multirow{2}{*}{$\begin{array}{l}\text { Activity } \\
\text { Range in } \\
\alpha \text {-curies } \\
\end{array}$} & \multirow{2}{*}{$\begin{array}{c}\text { Based on } \\
\text { WAC } \\
\% \text { RSD UL } \\
\text { for Precision } \\
\text { (15 } \\
\text { Replicates) } \\
\end{array}$} & \multirow{2}{*}{$\begin{array}{c}\text { Maximum } \\
\text { Allowable } \\
\% \text { RSD } \\
\left(95 \% \mathrm{CB}^{\mathrm{b}}\right. \\
\text { of UL) } \\
\end{array}$} & \multicolumn{2}{|c|}{$\begin{array}{c}\text { Criteria for Maximum Measured } \\
\text { PDP Precision (\%RSD) } \\
\text { (Six Replicates) }\end{array}$} & \multicolumn{2}{|c|}{$\begin{array}{c}\text { Criteria for Bias } \\
\text { (Values for } \% \mathrm{R}_{\mathrm{L}} \text { and } \% \mathrm{R}_{\mathrm{U}} \\
\text { for use in Equation } 11 \text { ) } \\
\text { (Six Replicates) }\end{array}$} \\
\hline & & & Non-interfering & Interfering & Non-interfering & Interfering \\
\hline $\begin{array}{c}>0 \text { to } \\
0.02\end{array}$ & 20 & 29.2 & 14.0 & 16 & $\begin{array}{l}\text { Lower: } 70 \\
\text { Upper: } 130\end{array}$ & $\begin{array}{l}\text { Lower: } 40 \\
\text { Upper: } 160\end{array}$ \\
\hline $\begin{array}{c}>0.02 \text { to } \\
0.2\end{array}$ & 15 & 21.9 & 10.5 & 12 & $\begin{array}{l}\text { Lower: } 70 \\
\text { Upper: } 130\end{array}$ & $\begin{array}{l}\text { Lower: } 40 \\
\text { Upper: } 160\end{array}$ \\
\hline $\begin{array}{c}>0.2 \text { to } \\
2.0\end{array}$ & 10 & 14.6 & 7.0 & 12 & $\begin{array}{l}\text { Lower: } 70 \\
\text { Upper: } 130\end{array}$ & $\begin{array}{l}\text { Lower: } 40 \\
\text { Upper: } 160\end{array}$ \\
\hline$>2.0$ & 5 & 7.3 & 3.5 & 6 & $\begin{array}{l}\text { Lower: } 70 \\
\text { Upper: } 130\end{array}$ & $\begin{array}{l}\text { Lower: } 40 \\
\text { Upper: } 160\end{array}$ \\
\hline
\end{tabular}

a - The 20\%RSD value for the low activity range was adopted from the WAC, A.3, Calibration Confirmation section. UL values for the remaining increased activity ranges were extrapolated on the basis of the capability of NDA systems to meet these limits.

b- Upper confidence bound for acceptable precision (expressed as \%RSD) at the $95 \%$ one-sided upper confidence based on 15 replicate measurements.

\section{Precision Criteria for Non-interfering Waste Matrices}

The true precision and bias of a measurement system are unknown. Estimates of the values of these parameters are acquired through the analysis of results of the total TRU $\alpha$-activity parameter from a set of six replicate assay measurements of the same NDA PDP sample. The more measurements acquired, the better are the precision and bias estimates of the NDA system for a given NDA PDP sample configuration.

The NDA PDP limits for measured precision of a non-interfering type matrix, determined from six replicate samples, are given in Table D-1. The values for six replicate measurements were derived from the upper confidence bounds for 15 replicate measurements. The derivation results in a downward adjustment of the acceptable measured precision values compared to that allowable for 15 replicate measurements, as shown in column 2.

For example, when six replicates are used, a measured value of $18 \%$ for the RSD of an assay system in the low activity range, even though less than the $20 \%$ allowable using 15 replicates, does not mean the implicit limit of an upper confidence bound of $29.2 \%$ has been met. In fact, the $95 \%$ one-sided upper confidence bound for this six-replicate example is approximately $38 \%$ - considerably higher than the allowable limit. Hence, the allowable measured precision with only six replicates is lower than that for 15 replicates for each $\alpha$-activity range.

Since the $95 \%$ confidence limit for relative standard deviation depends only on the standard deviation itself, it is possible, with a pre-specified fixed sample size, to determine ahead of time exactly how large the calculated NDA PDP precision-point estimate value can be and still have an associated upper onesided 95\% confidence limit that meets the criteria in Table D-1. The fourth and fifth columns of Table D-1 tabulate these maximum measured relative precision point estimate values using six replicates. The limits in columns 5 and 6 are used to compare the calculated NDA measurement system point estimate for relative standard deviation from six replicate measurements on non-interfering and interfering NDA PDP sample matrices, respectively. (Exactly how the values for interfering matrices were obtained is described below.) Note that comparing the non-interfering NDA measurement system point estimate to the value in column 4 is equivalent to comparing the associated upper one-sided $95 \%$ confidence limit to the value in column 3 . That is, an NDA PDP point estimate of the value indicated in column 4 using six replicates will have a $95 \%$ upper one-sided confidence limit equal to the value in column 3 . 


\section{Calculating Limits for Measured Relative Precision}

The limits specified in column 4 for the non-interfering PDP sample relative precision (standard deviation divided by the known value) are derived from confidence interval calculations for the variance (i.e., the square of the standard deviation) of a distribution. The derivation is described below.

\section{General Derivation}

Let $\sigma^{2}$ equal the true variance and let $1-\alpha$ equal the desired confidence value. Furthermore, let $s^{2}$ equal the sample variance, and $\chi_{\alpha, n-1}^{2}$ equal the critical value of a chi-square distribution with $n-1$ degrees of freedom above which $\alpha \%$ of the distribution lies; that is, the critical value for the upper $\alpha \%$ tail of the distribution. Then, assuming a normal distribution, a two-sided $\alpha \%$ confidence interval for the true variance is (e.g., Anderson 1987).

$$
\frac{(n-1) s^{2}}{\chi_{\alpha / 2, n-1}^{2}}<\sigma^{2}<\frac{(n-1) s^{2}}{\chi_{1-\alpha / 2, n-1}^{2}}
$$

Based on this formula for the two-sided interval, the upper one-sided $(1-\alpha) \%$ confidence limit is

$$
\sigma^{2}<\frac{(n-1) s^{2}}{\chi_{1-\alpha, n-1}^{2}}
$$

and the corresponding upper limit for the true percent relative standard deviation is calculated as

$$
\frac{\sigma}{\mu_{0}} 100 \%<\sqrt{\frac{(n-1) \frac{s^{2}}{\mu_{0}^{2}}}{\chi_{1-\alpha, n-1}^{2}}} 100 \%
$$

where $\mu_{0}$ is the reference (or true) value of the NDA PDP sample.

For the NDA PDP tests, $n=6$ and $\chi_{1-\alpha, n-1}^{2}=\chi_{0.05,5}^{2}=1.145$ in Equation D-3. Substituting these values and the known value of the NDA PDP sample for $\mu_{0}$ in this formula gives an approximate upper onesided $95 \%$ confidence limit for the percent relative standard deviation for six replicates. If desired, this upper confidence limit can be directly compared to the numbers in column 3 of Table D-1 to determine if an assay system has met the relative precision criteria.

The numbers in column 4 of Table D-1 are derived by comparing the right portion of Equation D-3 to the appropriate number in column 3 of Table D-1 and solving for $s / \mu_{0}$. As an example, for the low activity range this calculation begins with the required inequality

$$
\sqrt{\frac{(n-1) \frac{s^{2}}{\mu_{0}^{2}}}{\chi_{1-\alpha, n-1}^{2}}} 100 \%<29.2 \%
$$


Solving for $s / \mu_{0}$ gives

$$
\frac{s}{\mu_{0}} 100 \%<\sqrt{\frac{(0.292)^{2} \chi_{1-\alpha, n-1}^{2}}{n-1}} 100 \%
$$

which, for six samples and 95\% confidence as specified in the PDP, gives

$$
\frac{s}{\mu_{0}} 100 \%<\sqrt{\frac{(0.292)^{2}(1.145)}{5}} 100 \%=14 \%
$$

Again, substituting the reference (or true) value of the NDA PDP sample for $\mu_{0}$ indicates that a calculated relative standard deviation of $14 \%$ or less meets the criterion for relative precision in the low activity range. Since the chi-square value and $n$ are the same for all activity levels, the column 4 values for the other activity levels are obtained simply by substituting the appropriate value in place of 0.292 in Equation D-6.

\section{Precision Criteria for Interfering Waste Matrices}

The WAC limits (section A.3, Calibration Confirmation) are specified for a "non-interfering matrix"; in other words, a waste matrix that does not have attributes that manifest themselves in the NDA measurement system as significant complicating error elements. To determine rational precision scoring criteria for the interfering type waste form, it was desirable to establish some relationship to program objectives that can be used as a basis for the NDA PDP criteria for the interfering matrix SWBs. There are certain program-defined limits for which assay systems are used to ensure compliance. In particular, there are the 200 fissile gram equivalent (FGE) material limits for 55-gallon containers and the TRU waste $\alpha$ activity definition used to discriminate TRU waste from low-level waste (LLW). At the high end, the precision of the assay system should be reasonable for waste containers approaching the $200 \mathrm{FGE}$ limit to ensure that an excessive number of SWBs do not exceed the limit at the $95 \%$ confidence level. Similarly, the waste assay system should be sufficiently precise for containers of low TRU mass loading (i.e., in the vicinity of the $100 \mathrm{nCi} / \mathrm{gram} \alpha$-activity criterion) to ensure that an unacceptable number of containers of TRU waste are not classified as LLW.

As a convenient base for determining precision criteria for interfering type waste matrix SWBs, the noninterfering compliance points in Table D-1 are used. For the low activity range, the nominal compliance point for meeting the WAC precision and bias criteria is $100 \mathrm{mg}$ of weapons-grade plutonium (WG $\mathrm{Pu}$ ). An acceptable assay system should be capable of detecting and quantifying TRU waste in 55-gallon waste containers at a level of $35 \mathrm{mg} \mathrm{WG} \mathrm{Pu}$, approximately $75 \mathrm{nCi} / \mathrm{g}$ waste at 100 pounds of waste. When assaying a container at the compliance point of $100 \mathrm{mg} \mathrm{WG} \mathrm{Pu}$, we would like to be sure at the $95 \%$ confidence level that the assay system will not return a value less than $35 \mathrm{mg} \mathrm{WG} \mathrm{Pu}$. This provides reasonable protection against classifying TRU waste as LLW. Based on this rationale, two standard deviations would correspond to $65 \mathrm{mg}(100 \mathrm{mg}-35 \mathrm{mg})$. One relative standard deviation would therefore be $32.5 \mathrm{mg} / 100 \mathrm{mg}$ or 0.325 . By substituting 0.325 in place of 0.292 in Equation D-6, we obtain a value of 0.155 (rounded up to 0.16 ) for the measured precision criterion for six replicate determinations of an interfering matrix SWB in the low activity range.

Using similar reasoning, a precision criterion can be assigned to the high-mass region. In this case, the nominal compliance point used is $160 \mathrm{~g} \mathrm{WG} \mathrm{Pu}$. When assaying a container at the compliance point of $160 \mathrm{~g} \mathrm{WG} \mathrm{Pu}$, we would like to be sure at the $95 \%$ confidence level that the assay system will not return a value greater than $200 \mathrm{~g} \mathrm{WG} \mathrm{Pu}$. This provides reasonable protection against mistakenly classifying a TRU waste SWB as not shippable when, in fact, it does not exceed the limit. Based on this rationale, two 
standard deviations would correspond to $40 \mathrm{~g}$ (200 g-160 g). One relative standard deviation would therefore be $20 \mathrm{~g} / 160 \mathrm{~g}$ or 0.125 . By substituting 0.125 in place of 0.292 in Equation D-6, we obtain a value of 0.0598 (rounded up to 0.06 ) for the measured precision criteria for six replicate determinations of an interfering matrix SWB in the high activity range.

No compelling programmatic objectives argue for specific precision limits for the low-middle and highmiddle ranges, although some thermal limits will fall into these ranges for some waste forms. Therefore, it was felt that arbitrary limits based on consistency and continuity in the use of the assay systems would be adequate for these ranges. The precision criteria for the low-middle and high-middle ranges were set at 0.12 for the RSD of six replicate determinations.

\section{D.3 Calculating Confidence Bounds For Bias}

The comparison of an assay system's performance to the bias requirements for the non-interfering and interfering NDA PDP samples requires calculation of the $95 \%$ two-sided confidence bounds for the true value, using the replicate measurement data set. Based on a $t$-distribution, the $(1-\alpha) \%$ two-sided confidence bounds for the true assay system mean are (assuming a normal distribution):

$$
\bar{x}-t_{1-\alpha / 2, n-1} \frac{s}{\sqrt{n}} \leq \mu_{0} \leq \bar{x}+t_{1-\alpha / 2, n-1} \frac{s}{\sqrt{n}}
$$

In terms of percent recovery, the bounds are

$$
\frac{\bar{x}-t_{1-\alpha / 2, n-1} \frac{s}{\sqrt{n}}}{\mu_{0}} \times 100 \%<100 \%<\frac{\bar{x}+t_{1-\alpha / 2, n-1} \frac{s}{\sqrt{n}}}{\mu_{0}} \times 100 \%,
$$

where $\mu_{0}$ is the known (or true) value. The lower and upper bounds, calculated per Equation E-8, must be greater than or equal to $\% R_{L}$ and less than or equal to $\% R_{U}$, respectively, where $\% R_{L}$ and $\% R_{U}$ are the appropriate lower and upper range-specific bounds from Table D-1. Equivalently, bounds for the point estimate, total TRU $\alpha$-activity percent recovery, can be obtained by solving the required inequalities for percent recovery. The required inequalities are:

$$
\frac{\bar{x}-t_{1-\alpha / 2, n-1} \frac{s}{\sqrt{n}}}{\mu_{0}} 100 \%>\% \mathrm{R}_{\mathrm{L}} \text { and } \frac{\bar{x}+t_{1-\alpha / 2, n-1} \frac{s}{\sqrt{n}}}{\mu_{0}} 100 \% \leq \% \mathrm{R}_{\mathrm{U}}
$$

which, on solving for percent recovery, gives

$$
\% \mathrm{R}_{\mathrm{L}}+\frac{t_{1-\alpha / 2, n-1} \frac{s}{\sqrt{n}}}{\mu_{0}} 100 \% \leq \frac{\bar{x}}{\mu_{0}} 100 \% \leq \% \mathrm{R}_{\mathrm{U}}-\frac{t_{1-\alpha / 2, n-1} \frac{s}{\sqrt{n}}}{\mu_{0}} 100 \%
$$

With six samples, $n=6$, and the corresponding $t$ value (for $95 \%$ two-sided confidence bounds) is 2.571 . Thus, the equation simplifies to:

$$
\% \mathrm{R}_{\mathrm{L}}+\frac{1.049 \mathrm{~s}}{\mu_{0}} 100 \%<\frac{\bar{x}}{\mu_{0}} 100 \%<\% \mathrm{R}_{\mathrm{U}}-\frac{1.049 \mathrm{~s}}{\mu_{0}} 100 \%
$$




\section{D.4 References}

Anderson, R. L. 1987, Practical Statistics for Analytical Chemists, New York, Van Nostrand Reinhold.

DOE/WIPP-02-3122, Transuranic Waste Acceptance Criteria for the Waste Isolation Pilot Plant, current revision, U.S. Department of Energy Waste Isolation Pilot Plant, Carlsbad, New Mexico. 


\section{Appendix E}

NDA PDP Standard Encapsulation Design 
This page intentionally blank 


\section{Appendix E NDA PDP Standard Encapsulation Design}

This appendix provides information on the NDA PDP standard encapsulation design used to contain the radioactive material/substrate mixture that constitutes a standard. Seven separate NDA PDP standard production phases were conducted at the Los Alamos National Laboratory. Each production phase was designed to yield a set of standards with specific attributes reflecting the DOE radioactive material inventory. All NDA PDP standards use a dual cylinder encapsulation design and have identical internal and external dimensions. The encapsulation cylinders are fabricated from seamless stainless steel tubing for all production phases except Phase III.A, which is fabricated from seamless zirconium tubing.

The PDP standard encapsulation is comprised of an inner and outer cylinder. The bottom end cap of each cylinder is laser-welded in place. The inner cylinder is then filled with the appropriate mixture of radioactive material and substrate. Once the mixture has been packed to a height specified in the production plan document (LAUR-96-2277, LAUR-98-213, LA-CP-00-54, LACP-00-110, LA-CP-01-208, LA-CP-03-0072, LA-CP-03-0763), a graphite-felt frit is installed, which presses the nuclear material/substrate assembly in place when the top cap is inserted and attached. The graphite frit also ensures that the radioactive material/substrate configuration does not change over time. The inner cylinder top cap is then welded in place using the tungsten inert gas (TIG) method. After the top cap is welded in place, the inner cylinder is inserted into the outer cylinder and the top outer cylinder cap is TIG-welded in place. An elevation view of both the inner and outer encapsulation cylinders is shown in Figure E-1. The final assembled NDA PDP standard encapsulation unit is shown in Figure E-2. Prepared assemblies of the stainless steel and zirconium dual encapsulation, complete with the substrate matrix (no radioactive material) and helium fill, have been tested in accordance with ANSI/HPS N43.6-1997 and comply with ANSI Classification 97C43323.

The dimensional and material properties of the NDA PDP standard were derived as a function of NDA PDP objectives, nondestructive waste assay system response characteristics, and practicalities of fabrication. A complete NDA PDP standard specification with supporting analyses is provided in the document, Performance Demonstration Program for Nondestructive Assay for the TRU Waste Characterization Program, Initial Cycle Source Design (INEL94/0104).

The as-specified PDP standard configuration complies with the following general requirements:

1. PDP standards must be physically stable and invariant with time in a defined geometry.

2. The PDP standard configuration must facilitate convenient loading of the standards into the PDP matrix drum or SWB.

3. The PDP standard dimensions must allow for the production of multiple-source spatial geometries within the PDP matrix drum or SWB.

4. The PDP standard encapsulation integrity must comply with all applicable standards and be acceptable for transportation to and storage at participating sites.

5. The PDP standard design must accommodate available fabrication technologies at a reasonable cost. 


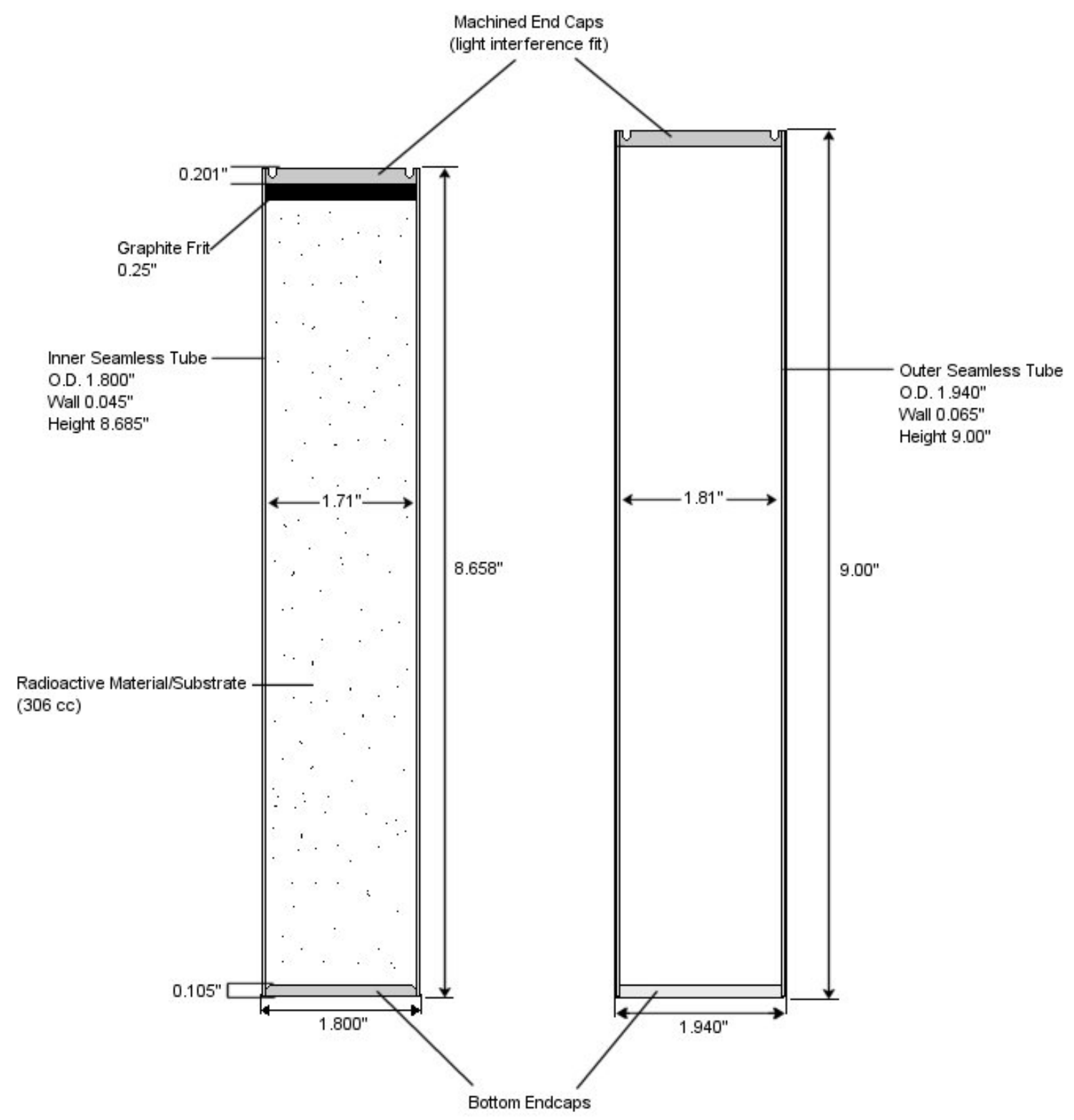

Figure E-1. PDP standard inner and outer cylinders, elevation view. 


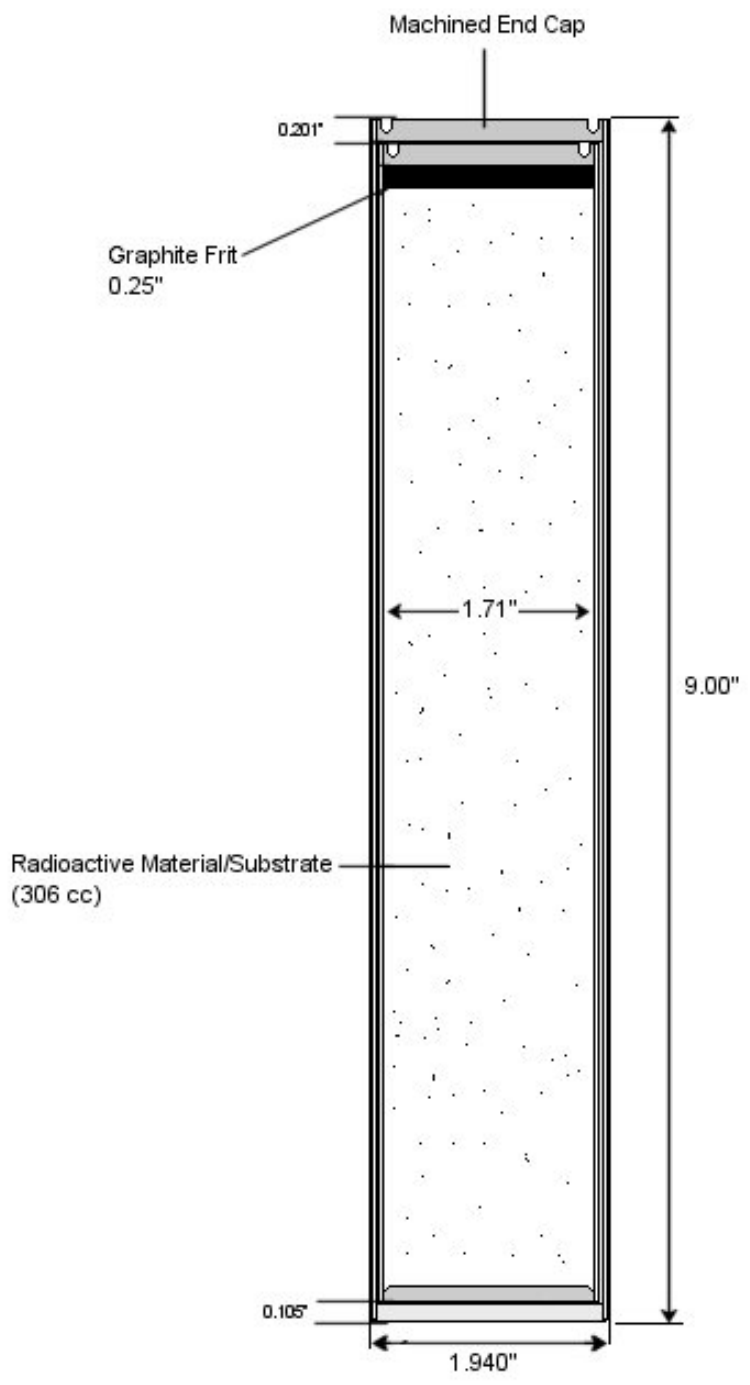

Figure E-2. PDP standard encapsulation assembly, elevation view. 
This page intentionally blank 


\section{Appendix F} NDA PDP Matrix SWB Specifications and Descriptions 
This page intentionally blank 


\section{Appendix $F$ NDA PDP Matrix SWB Specifications and Descriptions}

This appendix provides an overview of the NDA PDP matrix SWB set, general specifications, designs, and physical configurations. Additional technical detail on the design and fabrication of the NDA PDP matrix SWB set is provided in the following documents:

1. Nondestructive Assay Performance Demonstration Program: Standard Waste Box, Dry Combustibles Waste Matrix Surrogate-Specification, Design, and As-Built Material Properties for NDA PDP-SWB-01, INEEL/EXT-02-01271.

2. Nondestructive Assay Performance Demonstration Program: Standard Waste Box, Dry Combustibles Waste Matrix Surrogate-Specification, Design, and As-Built Material Properties for NDA PDP-SWB-02, INEEL/EXT-02-01272.

3. Nondestructive Assay Performance Demonstration Program: Standard Waste Box, Mixed Metals Waste Matrix Surrogate-Specification, Design, and As-Built Material Properties for NDA PDP-SWB-03, INEEL/EXT-02-01273.

4. Nondestructive Assay Performance Demonstration Program: Standard Waste Box, Mixed Metals Waste Matrix Surrogate-Specification, Design, and As-Built Material Properties for NDA PDP-SWB-04, INEEL/EXT-02-01274.

5. Nondestructive Assay Performance Demonstration Program: Standard Waste Box, Concrete Debris Waste Matrix Surrogate-Specification, Design, and As-Built Material Properties for NDA PDP-SWB-05, INEEL/EXT-03-00535.

6. Letter of Transfer of Materials to the NDA PDP from Washington TRU Solutions, LLC Central Characterization Project: CP:08:00361

7. Rocky Flats Environmental Technology Site Standard Waste Box Light Metals Waste Matrix Surrogate Specification, Design and Material Properties, CSM-013-00, March 2000, prepared by Custom Surrogate Matrices, Inc., Idaho Falls, ID for BNFL Instruments, Inc., Los Alamos, NM, under Subcontract Number: BII 99-304 (currently designated as NDA PDP SWB-07).

8. Rocky Flats Environmental Technology Site Standard Waste Box Moist Combustibles Waste Matrix Surrogate Specification, Design and Material Properties, CSM-011-00, March 2000, prepared by Custom Surrogate Matrices, Inc., Idaho Falls, ID for BNFL Instruments, Inc., Los Alamos, NM, under Subcontract Number: BII 99-304 (currently designated as NDA PDP SWB-06).

9. Idaho National Laboratory Central Characterization Facility Standard Waste Box Matrix Surrogate Inspection Summary Report, CSM-01-06, September 18, 2006, prepared by Custom Surrogate Matrices, Inc., Idaho Falls, ID, under P.O. WIPP1PO406914.

Use of the waste matrix box surrogate in combination with the NDA PDP standards provides the $\mathrm{CBFO}$ with information useful in assessing the ability of DOE facility measurement organizations to meet requirements for NDA of wastes intended for disposal at WIPP. The 
CBFO uses data generated in the NDA PDP as part of the assessment and approval process for measurement facilities supplying services for the characterization of WIPP TRU waste.

An NDA PDP matrix SWB must nominally represent the attributes and properties of DOEgenerated TRU wastes packaged in the SWB container. The NDA PDP SWB surrogate waste matrix characteristics considered in the specification and design of the NDA PDP SWB include matrix homogeneity, elemental composition, density, and total mass. The establishment of realistic attributes and design in terms of actual SWB matrix inventories allows CBFO to relate box assay system function and performance to WIPP waste matrix configurations. This allows statements regarding box assay system performance to be referenced to a specific, known, and documented boxed waste form configuration.

Multiple requirements define the design of the NDA PDP SWB waste matrix surrogate. These requirements range from ensuring a faithful replication of the nominal properties of the SWB waste types of interest to construction methods that result in robust long-lasting matrix modules and precise positioning of radioactive standards. The major matrix surrogate requirements are listed below.

1. Surrogate waste matrix properties must nominally represent specified DOE-generated waste forms as packaged in the SWB container. For the NDA Box PDP, three SWB matrix types were originally developed: mixed metals, dry combustibles, and concrete debris. These matrices were developed considering the expected properties of WIPP waste including matrix density, matrix elemental composition, matrix box fill height and geometrical distribution. Two additional matrix types have since been acquired into the PDP: light metals and wet combustibles (references 7 and 8 above).

2. The matrix surrogate array consists of 60 modules that can be used to construct variations in the physical geometry of the waste matrix ranging from uniform matrix loading to extreme heterogeneous distributions.

3. The surrogate matrix modules must be of manageable size and weight to support routine manual handling of the surrogate box module configuration.

4. The surrogate matrix modules must be robust to withstand transportation and routine handling.

5. The design of the sample configuration SWB must have an internal support structure that provides a convenient means to externally introduce and locate radioactive standards into the interior SWB volume containing matrix modules.

6. The SWB internal support structure must allow for positioning of one or more radioactive standards at various $\mathrm{X}-\mathrm{Y}$ box coordinates and vertical heights $(\mathrm{Z})$ sufficient to produce radioactivity distributions useful in the assessment of NDA performance.

7. The radioactive standard insertion mechanism must allow for precise and reproducible positioning of NDA PDP standards. 
8. The internal support structure design and materials must inherently minimize interferences arising from interaction with the characteristic radiations utilized for waste NDA measurements, be representative constituents, or have nearly equivalent properties of the waste form being simulated.

9. The matrix SWB design must allow consistent reproduction of matrix/standard configurations through time.

10. The matrix surrogate must include design provisions ensuring a stable, non-variable, and effectively inert matrix.

11. The matrix material must comply with health and safety considerations such that the materials are not hazardous, necessitating special handling and storage precautions.

12. The SWB surrogate must be readily distinguishable from the actual waste SWBs through a unique color and have a durable exterior alphanumeric identification.

13. A means to address void spaces introduced by surrogate configuration box fixtures must be provided.

14. Matrix modules must be of dimensions allowing interchangeability with matrix modules of different material type composition such as void, metals, or combustibles type modules.

15. The surrogate configuration shall not manifest systematic effects in commonly employed NDA measurement methods. Rather, the configuration must be random in attribute distribution.

In accordance with the general requirements listed above, the SWB waste matrix surrogate consists of a set of 60 modules that when combined, fill the interior volume of the SWB configuration box to a fill height of approximately $87 \%\left(1,538,744 \mathrm{~cm}^{3}\right)$ of the interior box height. The multiple module design allows flexibility in configuration of the NDA PDP SWB sample.

The multiple module strategy provides for sample configurations ranging from a uniform matrix distribution throughout the box to a heterogeneous distribution assembled with void modules or modules of differing matrix types. Presently, five NDA PDP SWB matrix types are available: mixed metals, light metals, dry combustibles, wet combustibles and concrete debris. The ability to mix and match matrix module types allows $\mathrm{CBFO}$ to create surrogate matrix configurations that span a range of test sample matrix configurations. An isometric of the SWB multi-module configuration as installed in an NDA PDP SWB configuration box is shown in figure E-1.

Each NDA PDP SWB matrix module set consists of two different module shapes, referred to as the center module and the end type module. The center modules are used in the rectangular midsection of the SWB box and the end modules are used to accommodate the curved ends of the SWB, as shown in figures E-2 and E-3, respectively.

The matrix modules have insert fixture pass-through holes to provide for the insertion and precise positioning of NDA PDP standards within the matrix. The SWB matrix module passthrough holes allow installation of aluminum NDA PDP standard insert fixtures for each of the 24 insert tube locations (figure E-4). If specified for a given matrix module, the NDA PDP 
standard(s) is positioned at a desired vertical location in the insert fixture through the use of locating pins. The insert fixture is then positioned into the SWB matrix module array. Matrix spacers are provided when appropriate, for use with the matrix surrogate to fill any void space within the insert fixture not occupied by NDA PDP standard(s), thus eliminating undesirable vertical void spaces. 


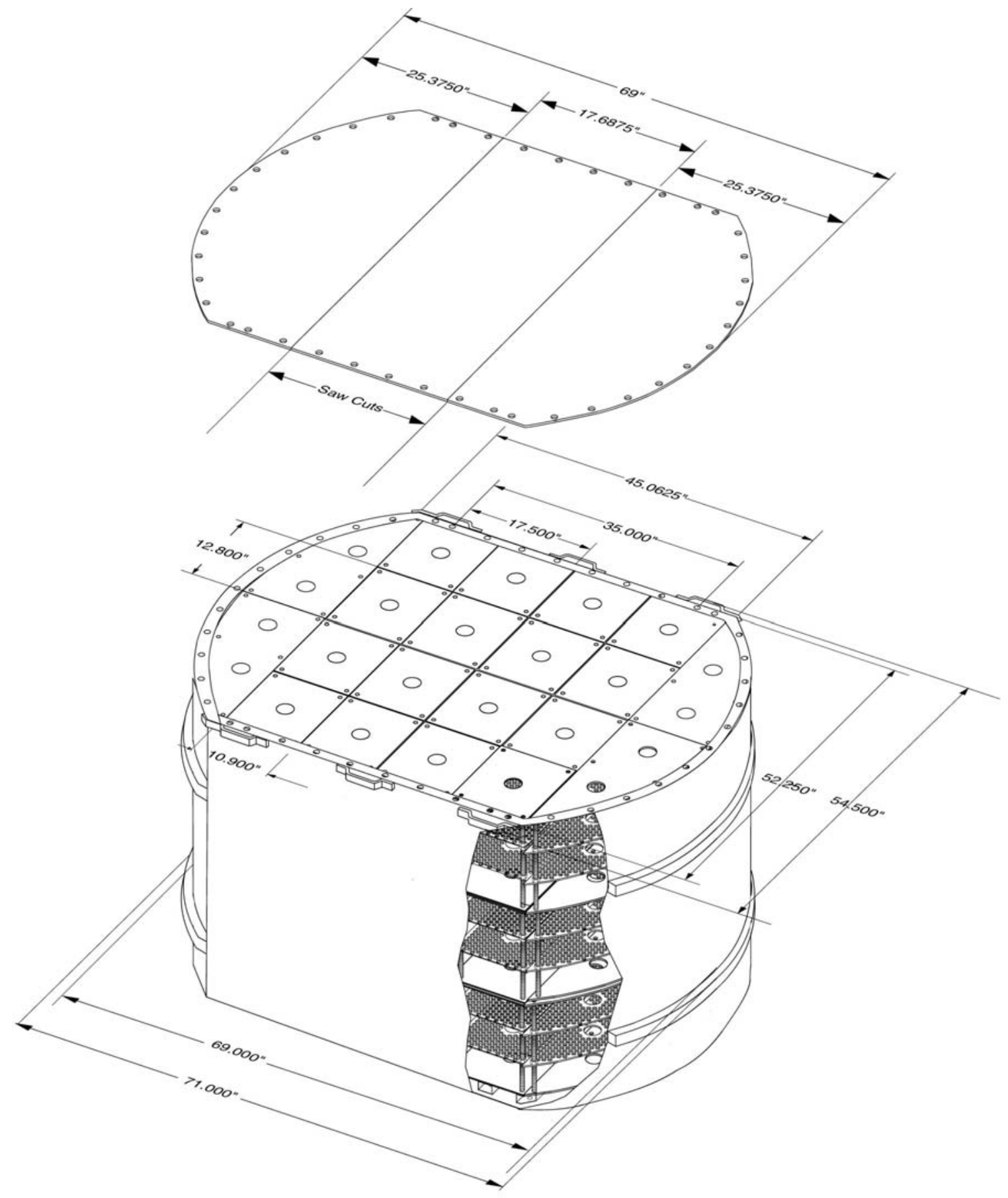

Figure F-1. Cutaway isometric of SWB matrix surrogate. 


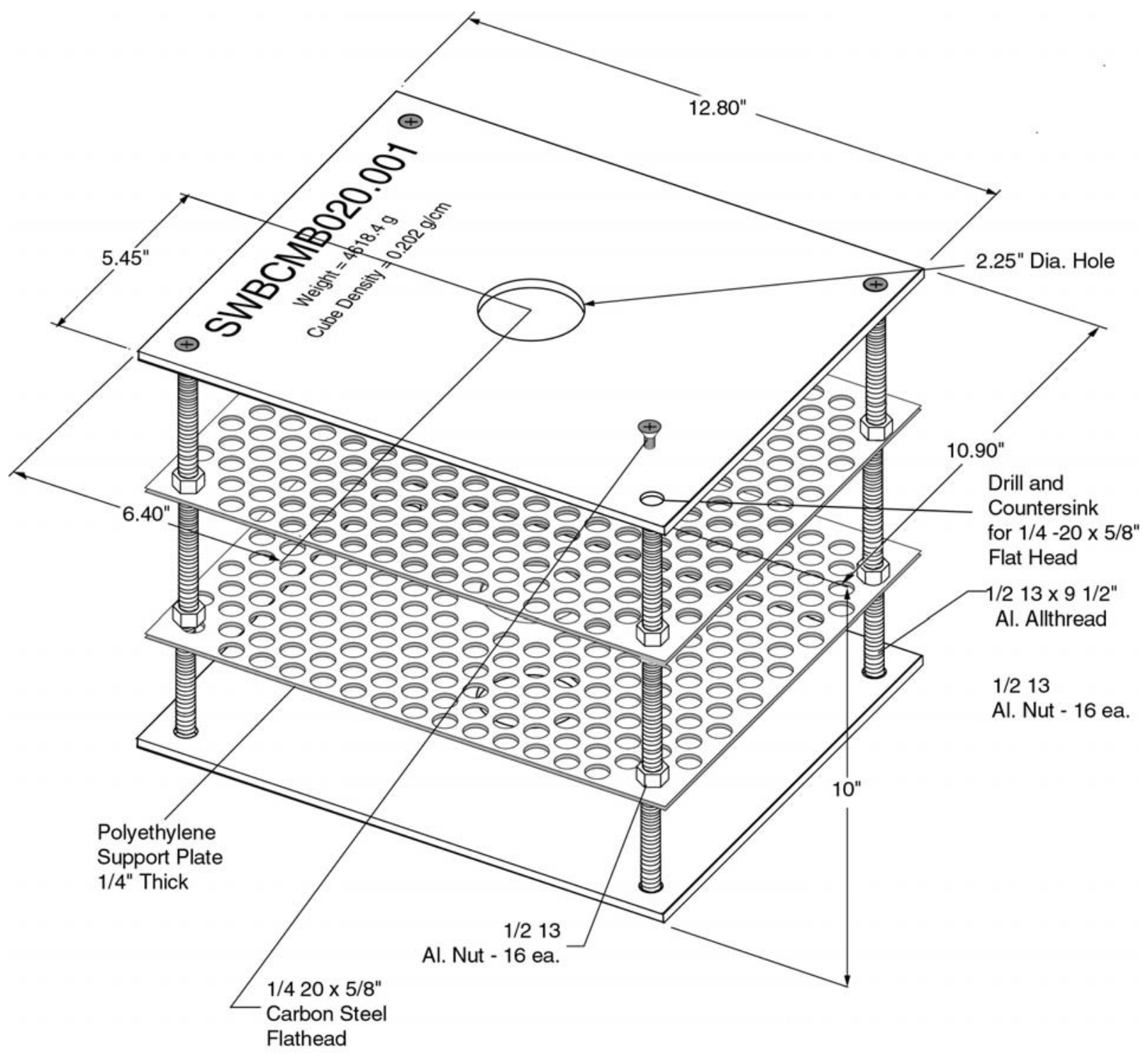

Figure F-2. Center type SWB matrix module. 


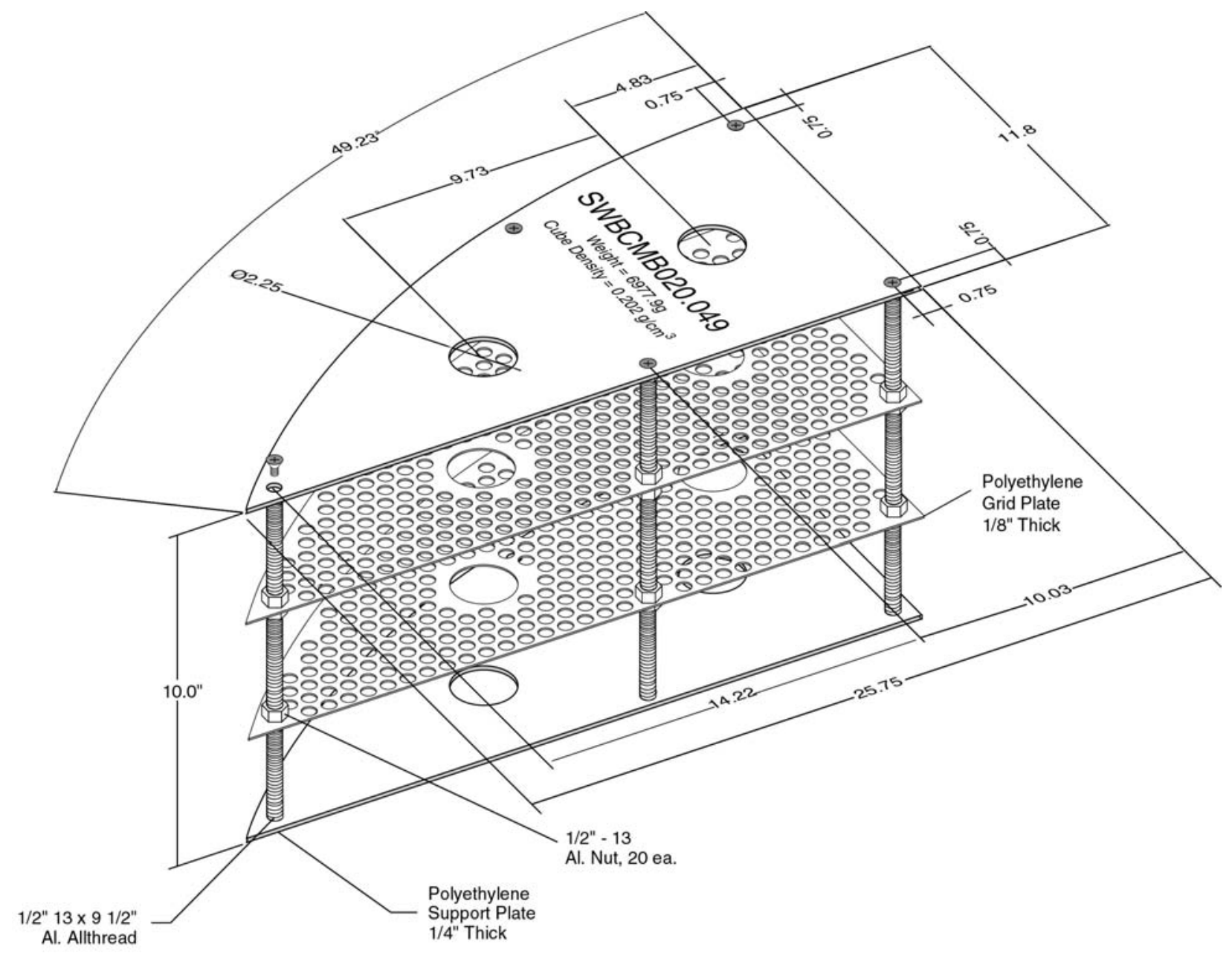

Figure F-3. End type SWB matrix module. 


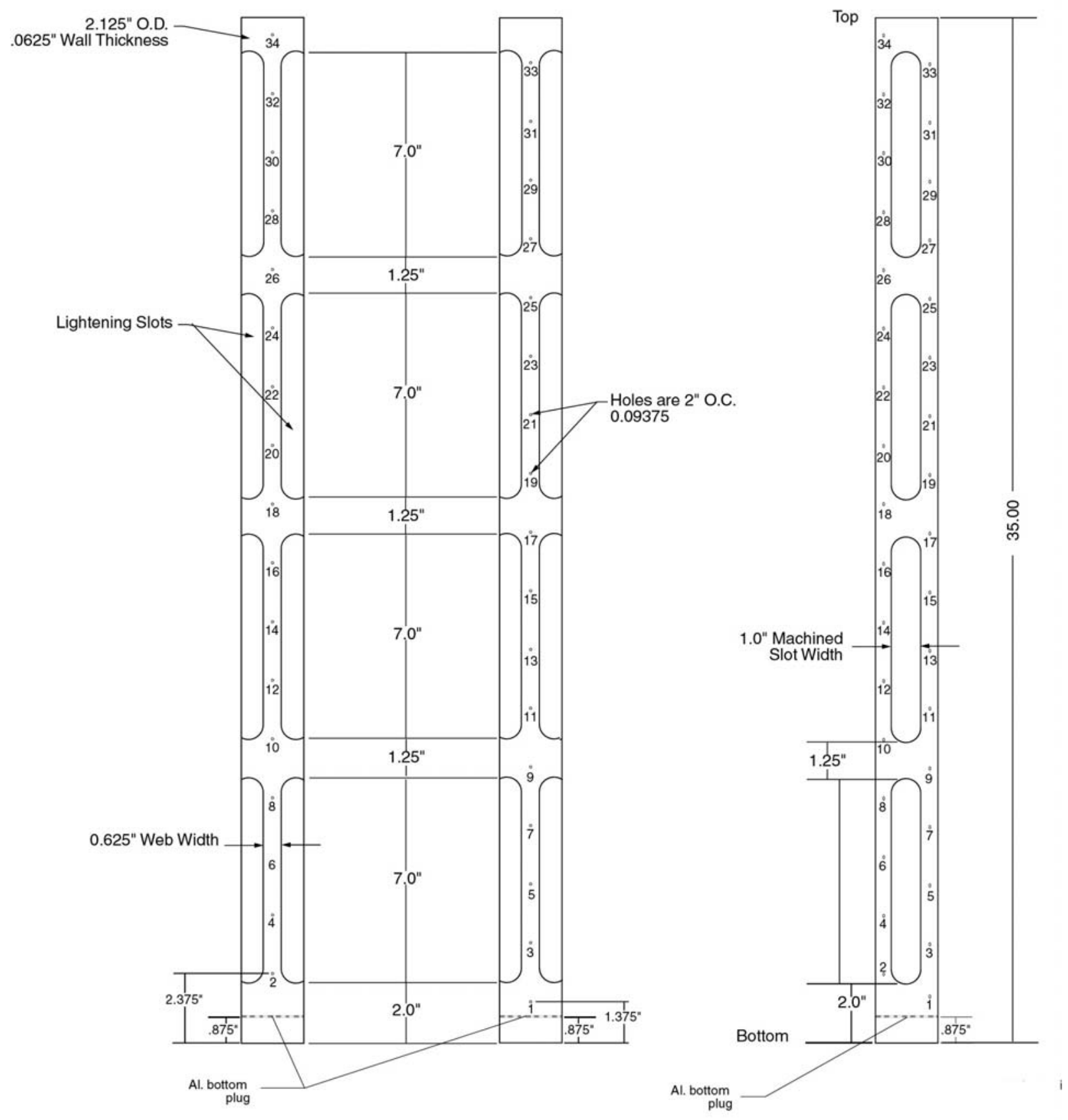

Figure F-4. SWB matrix surrogate PDP standard insert fixture. 
This page intentionally blank 